\title{
In Vitro Analog of Classical Conditioning of Feeding Behavior in Aplysia
}

\author{
Riccardo Mozzachiodi, Hilde A. Lechner, ${ }^{1}$ Douglas A. Baxter, and John H. Byrne ${ }^{2}$ \\ W.M. Keck Center for Neurobiology of Learning and Memory, Department of Neurobiology and Anatomy, \\ The University of Texas-Houston Medical School, Houston, Texas 77030, USA
}

\begin{abstract}
The feeding behavior of Aplysia californica can be classically conditioned using tactile stimulation of the lips as a conditioned stimulus (CS) and food as an unconditioned stimulus (US). Moreover, several neural correlates of classical conditioning have been identified. The present study extended previous work by developing an in vitro analog of classical conditioning and by investigating pairing-specific changes in neuronal and synaptic properties. The preparation consisted of the isolated cerebral and buccal ganglia. Electrical stimulation of a lip nerve $\left(\mathrm{AT}_{4}\right)$ and a branch of the esophageal nerve $\left(\mathrm{En}_{2}\right)$ served as the CS and US, respectively. Three protocols were used: paired, unpaired, and US alone. Only the paired protocol produced a significant increase in CS-evoked fictive feeding. At the cellular level, classical conditioning enhanced the magnitude of the CS-evoked synaptic input to pattern-initiating neuron B31/32. In addition, paired training enhanced both the magnitude of the CS-evoked synaptic input and the CS-evoked spike activity in command-like neuron CBI-2. The in vitro analog of classical conditioning reproduced all of the cellular changes that previously were identified following behavioral conditioning and has led to the identification of several new learning-related neural changes. In addition, the pairing-specific enhancement of the CS response in $\mathrm{CBI}-2$ indicates that some aspects of associative plasticity may occur at the level of the cerebral sensory neurons.
\end{abstract}

Although classical conditioning has been investigated in vertebrates (for reviews, see Thompson and Kim 1996; Rose 2000; Maren 2001; Welzl et al. 2001) and invertebrates (for reviews, see Carew and Sahley 1986; Byrne 1987; Hawkins et al. 1993; Glanzman 1995; Sahley and Crow 1998), most of the information available about the underlying cellular mechanisms has been obtained from studies that used aversive stimuli as unconditioned stimuli (US; e.g., Maksimova and Balaban 1984; Crow 1988; Moyer et al. 1996; Lamprecht et al. 1997; Dubnau and Tully 2001; Schafe et al. 2001) and from studies that focused on simple defensive reflexes (e.g., Carew et al. 1981; Hawkins et al. 1983, 1998; Walters and Byrne 1983; Buonomano and Byrne 1990; Sahley et al. 1994; Abrams et al. 1998; Antonov et al. 2001). In contrast, considerably less is known about the plasticity of appetitive forms of classical conditioning and about classical conditioning of relatively complex behaviors (e.g., Sahley et al. 1990; Menzel and Müller 1996; Colwill et al. 1997; Benjamin et al. 2000; Lechner et al. 2000a,b).

The feeding behavior of Aplysia californica has recently emerged as an attractive system for investigating the neuronal processes underlying the genesis of a complex behavior and the mechanisms of appetitive associative learning (Colwill et al. 1997; Nargeot et al. 1997, 1999a,b; Lechner et al. 2000a,b; Brembs et al. 2002; for review, see Elliott and Susswein 2002). The neural circuitry that generates the rhythmic activity associated with feeding behavior (central pattern generator, CPG) is located primarily in the buccal ganglia, and has been studied extensively. Furthermore, the isolated buccal ganglia retain the ability to express buccal motor patterns (BMPs) similar to those expressed in

'Present address: The Salk Institute for Biological Sciences, La Jolla, CA 92037, USA.

${ }^{2}$ Corresponding author.

E-MAIL John.H.Byrne@uth.tmc.edu; FAX (713) 500-0623.

Article and publication are at http://www.learnmem.org/cgi/doi/10.1101/ Im.65303. vivo (e.g., Susswein and Byrne 1988; Kirk 1989; Plummer and Kirk 1990; Morton and Chiel 1993a,b; Church and Lloyd 1994; Cropper and Weiss 1996; Nargeot et al. 1997; Evans and Cropper 1998; Kabotyanski et al. 2000; Jing and Weiss 2001, 2002; Sánchez and Kirk 2001), which are therefore often referred to as "fictive feeding."

Recently, Colwill et al. (1997) and Lechner et al. (2000a) classically conditioned feeding behavior in Aplysia using tactile stimulation of the lips as a conditioned stimulus (CS) and food as a US. Paired training produced a greater increase in the number of bites in response to the CS than unpaired training or presentation of the US alone. Lesion studies indicated that the anterior branch of the esophageal nerve $\left(\mathrm{En}_{2}\right)$ mediates the US (Lechner et al. 2000a). Furthermore, learning-induced changes in the CPG and cellular correlates in pattern-initiating neuron B31/32 were identified (Lechner et al. 2000b). Stimulation of the anterior tentacle nerve $\left(\mathrm{AT}_{4}\right)$, which conveys mechanosensory information about the CS, elicited a greater number of ingestion-like BMPs in ganglia from animals that had received paired training than in ganglia from unpaired animals. In addition, in preparations from animals that had received paired training, $\mathrm{AT}_{4}$ stimulation evoked a synaptic input in B31/32 of greater magnitude than in preparations from unpaired animals (Lechner et al. 2000b).

The goal of the present work was to develop a reduced preparation of the cerebral and buccal ganglia (Fig. 1A) suitable for studying classical conditioning of feeding behavior in vitro and to extend our previous analysis of learning-related changes in the feeding neural circuit. The isolated ganglia were trained with electrical stimulation of peripheral nerves $\mathrm{AT}_{4}$ (to mimic the $\mathrm{CS}$ ) and $\mathrm{En}_{2}$ (to mimic the US; Fig. 1A). Paired presentation of the CS and US in vitro produced plastic changes similar to those following classical conditioning in vivo (i.e., increases in CSevoked fictive feeding and the CS-evoked synaptic potentials in B31/32; Lechner et al. 2000b). In addition, the in vitro analog revealed a pairing-specific increase in both the CS-evoked synap- 
In Vitro Analog of Classical Conditioning

A.

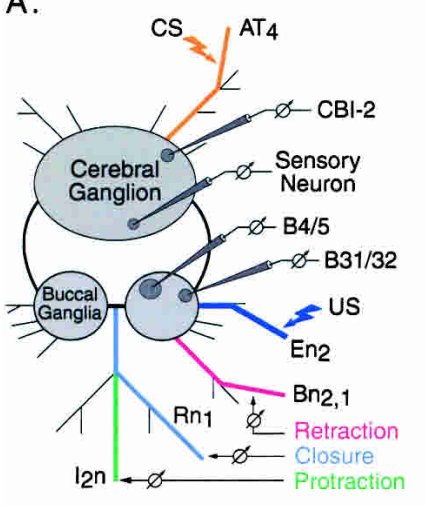

B.

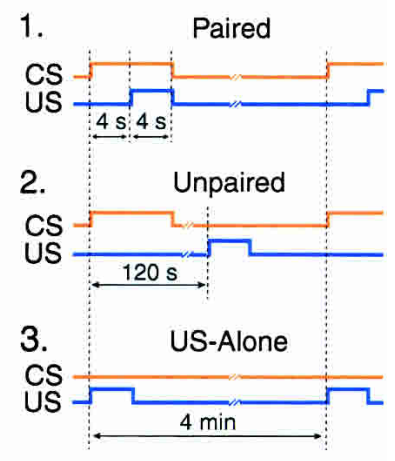

C.

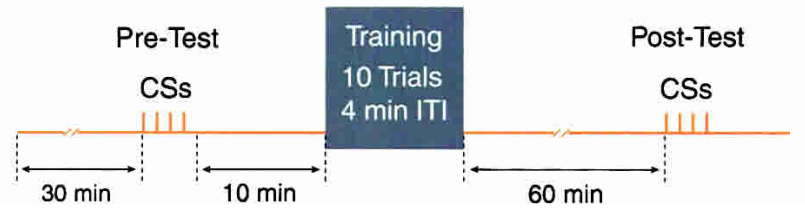

Figure 1 Schematic of the reduced preparation developed for in vitro classical conditioning. (A) The cerebral and buccal ganglia were isolated along with selected peripheral nerves. BMPs were recorded extracellularly from buccal nerves that innervate buccal muscles involved in protraction $\left(\mathrm{I}_{2} \mathrm{n}\right.$, green), closure $\left(\mathrm{Rn}_{1}\right.$, violet), and retraction $\left(\mathrm{Bn}_{2,1}\right.$, red) of the odontophore and radula. Stimulating electrodes were placed on nerves $\mathrm{AT}_{4}$ (orange) and $\mathrm{En}_{2}$ (dark blue). Electrical stimulation of $\mathrm{AT}_{4}(8 \mathrm{sec}, 5 \mathrm{~Hz}$, 0.5 -msec pulses) and $\mathrm{En}_{2}$ ( $4 \mathrm{sec}, 10 \mathrm{~Hz}, 0.5$-msec pulses) were used to mimic the CS (orange) and the US (dark blue), respectively. Intracellular recordings were made from $\mathrm{B} 31 / 32, \mathrm{~B} 4 / 5, \mathrm{CBI}-2$, and cerebral sensory neurons. $(B)$ Three protocols were used: paired [the CS onset preceded the US onset by $4 \mathrm{sec}$, and the CS and US overlapped for $4 \mathrm{sec}$ (B1)], unpaired [the CS and US did not overlap, and the ISI was $120 \mathrm{sec}(B 2)$ ], and US-alone [the CS was omitted $(B 3)]$. (C) Training procedure used for in vitro classical conditioning. After a 30-min baseline period, a Pre-Test, which consisted of four CSs, was performed. After a 10-min rest, one of the training protocols, which consisted of 10 trials with a 4-min ITI, was delivered. The preparations were allowed to rest for $60 \mathrm{~min}$, after which time four CSs were delivered (Post-Test). In the Pre-Test and Post-Test, the number of CS-evoked BMPs was counted during a 4-min period. In Experiments 2-5, measurements of the resting membrane potential, input resistance, response to the $\mathrm{CS}$ of $\mathrm{B} 31 / 32, \mathrm{~B} 4 / 5$, or $\mathrm{CBI} / 2$, as well as the strength of the synapse from $C B I-2$ to $B 31 / 32$ were made 10 min prior to the Pre-Test and Post-Test.

tic input to CBI-2 and the firing activity of CBI-2 in response to the CS. These results imply that classical conditioning strengthens the CS-evoked sensory input to specific neurons in the cerebral and buccal ganglia.

\section{RESULTS}

\section{The In Vitro Analog of Classical Conditioning Led to an Increase in the Number of CS-Evoked BMPs}

The goal of this study was to design an in vitro training procedure that closely resembled the in vivo training used by Lechner et al. (2000a). Electrical stimulation of peripheral nerves $\mathrm{AT}_{4}(8$ sec, $5 \mathrm{~Hz}, 0.5$-msec pulses) and $\mathrm{En}_{2}(4 \mathrm{sec}, 10 \mathrm{~Hz}, 0.5$-msec pulses) was used to mimic the CS and US, respectively (Fig. 1A; see Materials and Methods for details). To validate that electrical stimulation of $\mathrm{AT}_{4}$ reliably mimicked the tactile CS used in the behavioral experiments (i.e., stimulation of the lips; Lechner et al. $2000 \mathrm{a}, \mathrm{b})$, intracellular recordings were made from cerebral sensory neurons located in the $\mathrm{J}$ and $\mathrm{K}$ clusters, which are respon- sible for conveying tactile information from the lips (Rosen et al. $1979,1982)$, while $\mathrm{AT}_{4}$ was stimulated in a manner typically used for the in vitro CS. In all the preparations studied $(n=5)$, the in vitro CS elicited spike activity in neurons in the right $\mathrm{J}$ and $\mathrm{K}$ clusters, which can be classified as the cerebral mechanoafferents (Fig. 2). In addition, the frequency of firing of the sensory neurons closely matched the frequency of firing of the mechanoafferents in response to mechanical stimulation of the lips (Rosen et al. 1979).

The first experiment focused on the effects of in vitro classical conditioning on fictive feeding. The in vitro protocol is illustrated in Figure 1C (see also Materials and Methods). Prior to training, the number of CS-evoked BMPs elicited by the presentation of 4 CSs was counted during a 4-min Pre-Test. Then, each preparation was trained with one of the three protocols: paired presentation of the CS and US (Figs. 1B1, 3A), unpaired presentation of the CS and US (Figs. 1B2, 3B), or presentation of the US alone (Figs. 1B3, 3C). The training consisted of 10 trials with an intertrial interval (ITI) of $4 \mathrm{~min}$ (Fig. 1C). Then, $60 \mathrm{~min}$ after training, the Post-Test was performed and the number of CSevoked BMPs elicited by the presentation of four CSs was counted during a 4-min period. During the Pre-Test, the number of CSevoked BMPs did not differ significantly among the three training groups. For each preparation, the difference in the number of CS-evoked BMPs (i.e., the number of BMPs elicited by four CSs during the Post-Test minus the number of BMPs elicited by four CSs during the Pre-Test) was calculated. After training, the difference in the number of CS-evoked BMPs was greater in the paired group $(1.54 \pm 0.33 \mathrm{BMPs}, n=13)$ as compared with the unpaired group $(0.46 \pm 0.29 \mathrm{BMPs}, n=13)$ or the US-alone group $\left(0.31 \pm 0.29\right.$ BMPs, $n=13 ; H_{2}=9.423, p<0.05$; Fig. $\left.4 \mathrm{~A}\right)$. Post hoc analysis revealed a significant difference between the paired and the unpaired groups $(q=4.587, p<0.05)$ and between the paired and the US-alone groups $(q=3.71, p<0.05)$, whereas no significant difference was detected between the unpaired and the US-alone groups $(q=0.943, p \geq 0.05)$. These results indicate that the increased ability of the CS to evoke BMPs after training was specifically related to the paired association between CS and US.

\section{Paired Training Did Not Produce an Associative Change in the Occurrence of Spontaneous BMPs}

The increase in the number of BMPs produced by paired training could be due to a rise in the spontaneous activity in the feeding

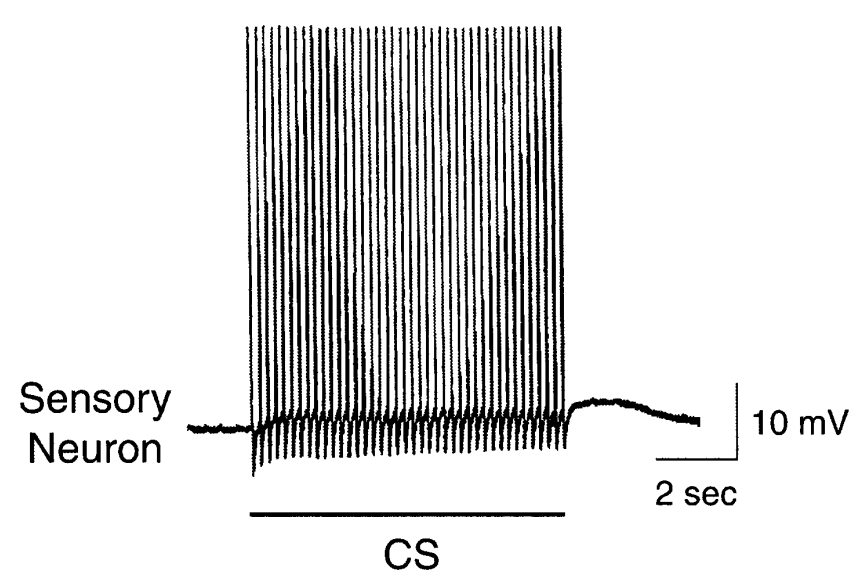

Figure 2 Response of a cerebral sensory neuron to the CS analog. Electrical stimulation of $\mathrm{AT}_{4}(8 \mathrm{sec}, 5 \mathrm{~Hz}, 0.5$-msec pulses, $8.5 \mathrm{~V})$, which was used to mimic the CS, elicited a train of action potentials in the cerebral sensory neuron located in the ipsilateral J cluster. 


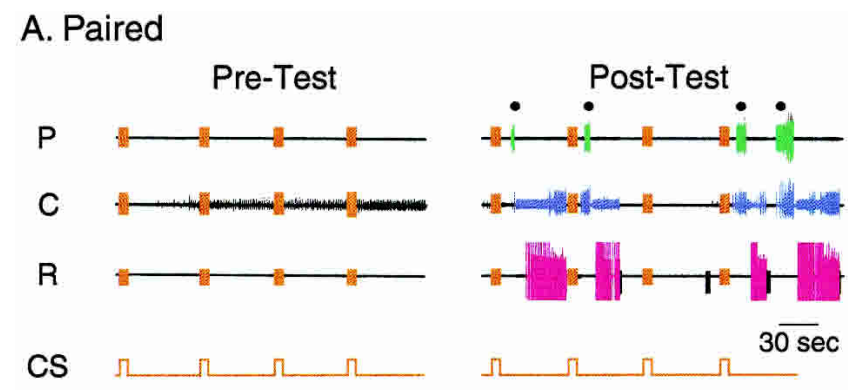

B. Unpaired
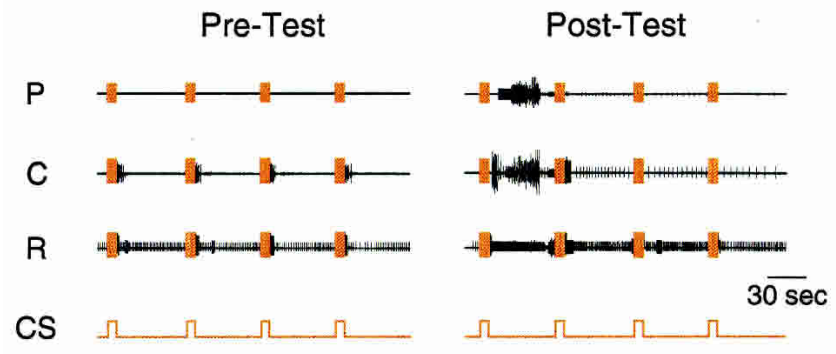

C. US-Alone
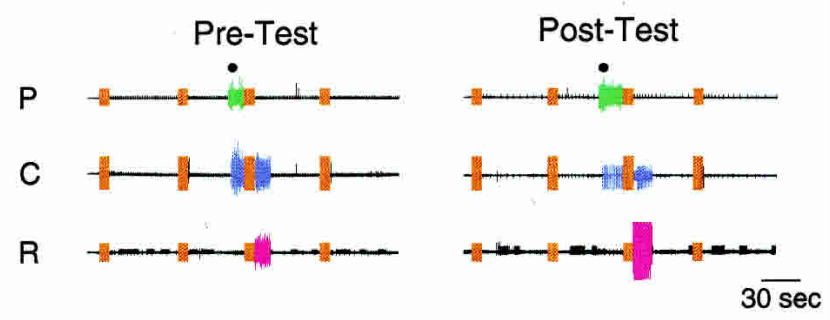

CS

Figure 3 Ability of the CS to elicit BMPs before and after in vitro training procedures. Paired $(A)$, unpaired $(B)$, or US-alone $(C)$ protocols were used. The number of CS-elicited BMPs was determined before (Pre-Test) and after (Post-Test) the training by four presentations of the CS, with an ISI of $1 \mathrm{~min}$ (traces in orange in $A, B$, and C). BMPs consisted of trains of large-unit activity recorded from buccal nerves $\mathrm{I}_{2} \mathrm{n}$ (protraction, $\mathrm{P}$ ), $\mathrm{Rn}_{1}$ (closure, C), and $\mathrm{Bn}_{2,1}$ (retraction, R). Examples of extracellular recordings are illustrated. Artifacts (orange) due to electrical stimulation of $\mathrm{AT}_{4}$ are present during each presentation of the CS. The filled circles placed over the $\mathrm{I}_{2} \mathrm{n}$ recording (i.e., $\mathrm{P}$ ) indicate the occurrences of CS-evoked BMPs. During the occurrence of a BMP, activity during $P, C$, and $R$ is represented in green, violet, and red, respectively. Note that the pattern occurring during the Post-Test in panel $B$ was incomplete because of the lack of large-unit activity during the retraction phase and therefore was not classified as a BMP and was not included in the counting of the CS-elicited BMPs (see Materials and Methods). Also, note that the four CSs were presented during the Pre-Test and Post-Test regardless of the occurrence of a BMP.

CPG, rather than a pairing-specific enhancement of the response to the CS. Therefore, the occurrence of spontaneous BMPs was measured before and after training. The frequencies of spontaneous BMPs (i.e., the number of BMPs per minute) occurring before (i.e., during a 30-min baseline period prior to the Pre-Test) and after (i.e., during a 60-min resting period) training were calculated for each preparation. Before training, the mean frequencies of spontaneous BMPs in the paired, unpaired, and US-alone groups were not significantly different. Changes in the frequency of spontaneous BMPs during the 60-min resting period after training were expressed as a percentage of the fre- quency measured during the 30-min baseline (i.e., percent of baseline). Overall, spontaneous activity decreased slightly in all groups over the course of the experiment. However, these changes were similar for the paired $(89.3 \% \pm 36.2 \%$ of Pre-Test; $n=13)$, unpaired $(84.7 \% \pm 23.3 \%$ of Pre-Test; $n=13)$, and US-alone $(76.7 \% \pm 19.4 \%$ of Pre-Test; $n=13)$ groups $\left(H_{2}=0.134\right.$, $p=0.94$; Fig. 4B). These results indicate that in vitro paired training appeared to selectively enhance the response to the CS, without affecting the baseline activity of the feeding CPG.

\section{Paired Training Selectively Increased the Number of Ingestion-like BMPs}

In Aplysia, BMPs have been recorded in vivo and correlated with behavioral ingestion and rejection (Cropper et al. 1990; Morton and Chiel 1993a,b; Hurwitz et al. 1996). A key feature for distinguishing between neural activity that is associated with ingestion and neural activity that is associated with rejection is the relative overlap between activity in nerves that control radula closure (i.e., large-unit activity in $\mathrm{Rn}_{1}$ ) and activity in nerves that control the protraction or retraction of the radula and odontophore (i.e., large-unit activity in $\mathrm{I}_{2} \mathrm{n}$ and $\mathrm{Bn}_{2,1}$, respectively). Because these phase relationships are maintained in vitro (e.g., Morton and Chiel 1993b; Nargeot et al. 1997), BMPs can be classified as ingestion-like (Fig. 5A) or rejection-like (Fig. 5B) according to the criteria described in Materials and Methods (see also Morton and Chiel 1993a,b; Nargeot et al. 1997). These criteria were used to classify the BMPs evoked by the CS. Because of the very low number of CS-evoked BMPs produced during the Pre-Test in paired (0.15 \pm 0.15 BMPs; $n=13)$, unpaired (0.15 \pm 0.1 BMPs; $n=13)$, and US-alone ( $0.31 \pm 0.18$ BMPs; $n=13)$ groups, we focused on the CS-evoked BMPs elicited during the Post-Test.

The number of CS-evoked ingestion-like BMPs during the Post-Test was greater in the paired group $(1.08 \pm 0.27 \mathrm{BMPs}$; $n=13)$ as compared with the unpaired group (0.23 \pm 0.17 BMPs; $n=13)$ or the US-alone group $(0.15 \pm 0.1$ BMPs, $n=13$; $H_{2}=16.064, p<0.05$; Fig. 6$)$. Post hoc analysis revealed a significant difference between the paired and the unpaired groups $(q=4.95, p<0.05)$ and between the paired and the US-alone groups $(q=4.269, p<0.05)$, whereas no significant difference was detected between the unpaired and the US-alone groups $(q=1.414, p \geq 0.05)$. In contrast, the number of rejection-like BMPs measured during the Post-Test did not differ between the paired $(0.31 \pm 0.13$ BMPs; $n=13)$, the unpaired $(0.23 \pm 0.12$ BMPs; $n=13)$, and the US-alone $(0.23 \pm 0.17$ BMPs; $n=13)$ groups $\left(H_{2}=2.145, p=0.342\right.$; Fig. 6$)$. Similarly, the number of other BMPs measured during the Post-Test did not differ between the paired $(0.31 \pm 0.24 \mathrm{BMPs} ; n=13)$, the unpaired $(0.15 \pm 0.1$ BMPs; $n=13)$, and the US-alone $(0.31 \pm 0.13$ BMPs; $n=13)$ groups $\left(H_{2}=1.413, p=0.493\right.$; Fig. 6). Importantly, Lechner et al. (2000b) reported that an increase in the number of CS-evoked ingestion-like BMPs also occurred following in vivo classical conditioning.

In summary, these results indicate that in vitro paired stimulation of peripheral nerves induced pairing-specific changes in the neural circuitry that mediates feeding behavior. These changes resulted in an increased number of CS-evoked ingestion-like BMPs. In addition, these changes were not due to an increased spontaneous activity of the CPG, but were specific to the activation of the CS pathway.

\section{Paired Training Enhanced the CS-Evoked Synaptic Input to $\mathrm{B} 31 / 32$}

The results described above indicate that the neural circuitry that mediates feeding can be trained in vitro with a classical conditioning protocol, which results in an increase in the number of 
In Vitro Analog of Classical Conditioning

A.

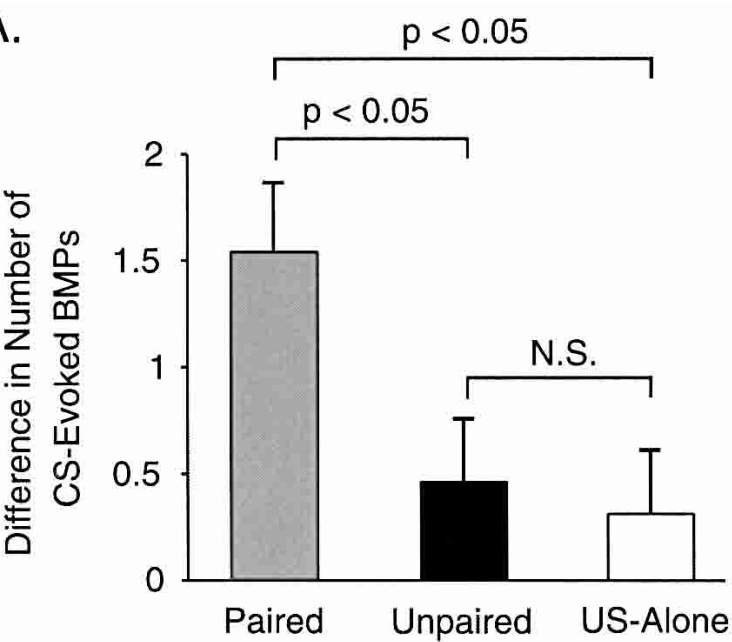

B.

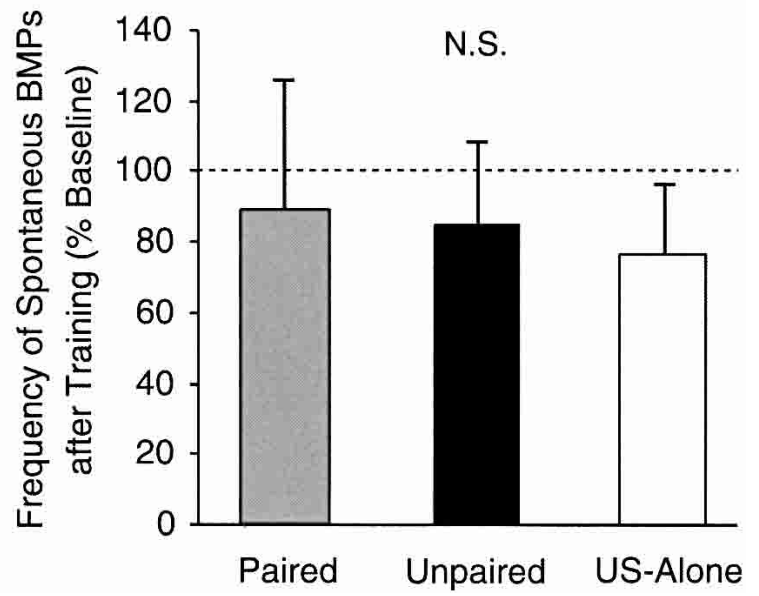

Figure 4 Analysis of the changes in the CS-elicited and spontaneous activity in the CPG following paired (gray), unpaired (black), or US-alone (white) training protocols. $(A)$ The effectiveness of classical conditioning was assessed by determining the difference in the number of CS-evoked BMPs (i.e., the number of CS-evoked BMPs during the Post-Test minus the number of CS-evoked BMPs during the Pre-Test). In this and subsequent illustrations, cumulative data are displayed as mean \pm SEM. The level of significance was set at $p<0.05$; (N.S.) the difference was not significant. Paired training resulted in a significantly greater difference in the number of CS-elicited BMPs as compared with either unpaired training or US-alone presentation. ( $B$ ) The frequency of spontaneously occurring BMPs (i.e., the number of BMPs per minute) did not change after training in paired, unpaired, or US-alone groups of preparations. Thus, pairing-specific plasticity induced by classical conditioning was specifically associated with the CS and was not manifest as an increased baseline activity of the feeding CPG.

CS-evoked ingestion-like BMPs. We next attempted to identify specific cellular correlates that could account for these changes. Activity in neuron B31/32 initiates BMPs, and hyperpolarizing B31/32 can prevent the expression of BMPs, which indicate that B31/32 plays a pivotal role in the expression of BMPs (Susswein and Byrne 1988; Hurwitz et al. 1994, 1996). In addition, in vivo classical conditioning produced an enhancement of the CSevoked synaptic input to B31/32 (Lechner et al. 2000b). Thus, B31/32 was a reasonable candidate for mediating the pairingspecific changes. The properties of B31/32 were examined in gan- glia trained with either the paired or unpaired protocol. Because the previous experiment found no effect of the US-alone training (Figs. 4, 6), the US-alone group was not included in the following set of experiments. The training procedure was identical to that used in the previous series of experiments except that the right buccal ganglion was desheathed to expose neuron B31/32.

Before training, the number of CS-evoked BMPs did not differ significantly between the paired and the unpaired groups. As in the previous experiment, paired training resulted in a significantly greater difference in the number of CS-evoked BMPs (1.9 \pm 0.43 BMPs; $n=10)$ than unpaired training $(0.27 \pm 0.47$ BMPs; $n=11 ; U=24.5, p<0.05) 60$ min after in vitro conditioning.

To examine whether classical conditioning affected the strength of the CS-evoked synaptic input to B31/32, the magnitude of the CS-evoked cPSP in B31/32 was measured 10 min prior to the Pre-Test and the Post-Test. The peak amplitude over the 8 -sec duration of the CS was measured. The amplitude of the cPSP before training did not differ between the paired and the unpaired groups (Fig. 7A1,B1). The increase of the peak amplitude of the CS-evoked cPSP was significantly greater after paired $(245.5 \% \pm 41.4 \%$ of Pre-Test; $n=10)$ than after unpaired $(147.6 \% \pm 18.2 \%$ of Pre-Test; $n=11)$ training $(U=26, p<0.05$; Fig. 7A2,B2,C). We also analyzed the net depolarization (i.e., the area of the cPSP) over the 8-sec duration of the CS. The area of the cPSPs did not differ between the paired and the unpaired groups (Fig. 7A1,B1) before training. The increase in the area of the CS-evoked cPSP was significantly greater after paired $(283 \% \pm 56.5 \%$ of Pre-Test; $n=10)$ than after unpaired $(128.4 \% \pm 20.9 \%$ of Pre-Test; $n=11)$ training $(U=22, p<0.05$; Fig. 7A2,B2,D).

These results indicate that the net excitatory input to pattern-initiating neuron B31/32 increased after paired training. Interestingly, a significant positive correlation was detected between the change in the peak amplitude of the CS-evoked cPSP in B31/32 and the difference in the number of CS-evoked BMPs $(r=0.45, p<0.05)$. This result indicates that the strengthening of the CS-evoked input to B31/32 may functionally contribute to the increase in the number of CS-evoked BMPs. Importantly, the enhanced synaptic input to B31/32 produced by paired training in vitro was very similar (for both the peak amplitude and the area of cPSP) to that measured in buccal ganglia isolated from animals classically conditioned in vivo (Lechner et al. 2000b). This similarity indicates that in vivo and in vitro classical conditioning could share common mechanisms.

\section{Paired Training Did Not Produce an Associative Change} in the Intrinsic Properties of $B 31 / 32$

Two of the intrinsic properties of B31/32 (i.e., the resting membrane potential and input resistance) were examined before and after training. During the Pre-Test, the average resting membrane potential of B31/32 in the paired $(-65.2 \pm 1.1 \mathrm{mV} ; n=10)$ and unpaired groups $(-66.8 \pm 1.1 \mathrm{mV} ; n=10)$ did not differ significantly $(U=33, p=0.212)$. Moreover, the resting membrane potential of B31/32 did not change after either paired or unpaired training (Table 1). The average input resistance of $\mathrm{B} 31 / 32$ in the paired $(2.6 \pm 0.2 \mathrm{M} \Omega ; n=10)$ and unpaired $(3.1 \pm 0.2 \mathrm{M} \Omega$; $n=10)$ groups was not significantly different before training $(U=28, p=0.104)$. Although in both groups the input resistance was reduced by $\sim 15 \%$ after training, the change in the input resistance of B31/32 after training in the paired and unpaired groups did not differ significantly (Table 1), at least to the extent to which such a change could be detected in the soma. A similar result (i.e., the lack of effects of training on the intrinsic properties of B31/32) was also observed following behavioral training (Lechner et al. 2000b). 


\section{A. Ingestion-Like}

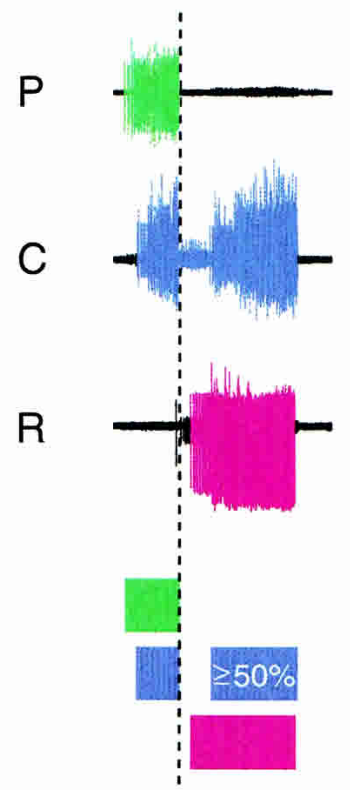

B. Rejection-Like

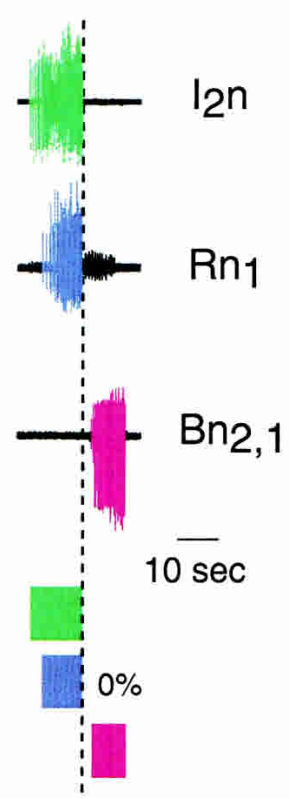

Figure 5 Classification of the BMPs expressed by the CPG. BMPs were classified as ingestion-like $(A)$ or rejection-like BMPs $(B)$ based on the relative overlap of closure activity in the protraction/retraction cycle. The relative duration of large-unit activity for $\mathrm{P}$ (green), $\mathrm{C}$ (violet), and $\mathrm{R}$ (red) is diagrammed by shaded boxes underneath the recorded traces. $(A)$ BMPs were classified as ingestion-like if $\geq 50 \%$ of large-unit activity of $R n_{1}$ occurred after the end of large-unit activity of $\mathrm{I}_{2} \mathrm{n}$ (dashed line). (B) BMPs were classified as rejection-like if there was no overlap between large-unit activity of $\mathrm{Rn}_{1}$ and large-unit activity of $B n_{2,1}$. The examples shown in $A$ and $B$ were BMPs spontaneously expressed in the same preparation.

Together these results indicate that paired training produced a potentiation of the CS-evoked synaptic input to B31/32 that persisted for at least $1 \mathrm{~h}$ after the training. However, paired training did not induce any apparent change in either the resting membrane potential or input resistance of B31/32. Because activation of B31/32 appears to be critical in the expression of patterned activity in the feeding CPG (Susswein and Byrne 1988), it is likely that the increased synaptic input to B31/32 contributes to the greater number of BMPs evoked by the CS after paired training.

\section{Paired Training Did Not Produce an Associative Change in the Spike Activity, Synaptic Input, or Intrinsic Properties of Neuron B4/5}

In addition to monitoring CS-evoked BMPs and cPSPs in B31/32, we also examined whether B4/5 was a site of plasticity associated with in vitro classical conditioning. B4/5 is a multifunctional neuron of the feeding CPG (Gardner 1971, 1977), which is mainly active during the retraction phase of a BMP (Church and Lloyd 1994; Warman and Chiel 1995; Jing and Weiss 2001). B4/5 receives excitatory input from cerebral mechanoafferents (Rosen et al. 1982) as well as from command-like interneurons such as CBI-1 (Rosen et al. 1991). In addition, B4/5 is involved in the neural mechanisms of motor program switching (Warman and Chiel 1995; Kabotyanski et al. 1997, 1998; Jing and Weiss 2001). We examined whether B4/5 was a site of associative plasticity. Training procedures were identical to those used in the previous experiments.

Before training, the number of CS-evoked BMPs elicited during the Pre-Test did not differ significantly between the paired

and the unpaired groups. As in the previous experiments, paired training resulted in a significantly greater increase in the number of CS-evoked BMPs $(1.39 \pm 0.29$ BMPs; $n=13)$ than unpaired training $(0.08 \pm 0.24$ BMPs; $n=13) 60 \mathrm{~min}$ after conditioning $(U=30.5, p<0.05)$.

We first analyzed whether in vitro classical conditioning affected the ability of the CS pathway to activate B4/5. B4/5 exhibited a train of action potentials in response to the CS (see Materials and Methods). When the CS evoked a BMP (mostly during the Post-Test after paired training), B4/5 exhibited a second train of action potentials predominantly during the retraction phase. However, the contribution of the train in B4/5 during BMPs was not included in the analysis of the effect of training on the ability of the CS to activate B4/5. Therefore, only the action potentials elicited in B4/5 during the four CSs in Pre-Test and Post-Test were analyzed (see also Materials and Methods). To assess the level of spike activity of $\mathrm{B} / 45$ in response to the CS prior to training, the number of action potentials elicited in $\mathrm{B} 4 / 5$ during the Pre-Test was counted. The total number of action potentials evoked in B4/5 by four CSs during the Pre-Test did not differ between the paired and the unpaired groups $(U=62.5$, $p=0.901)$. Thus, these data were pooled and the overall average number of action potentials elicited in B4/5 during the Pre-Test was $209 \pm 33.3(n=23)$. This procedure provided an estimate of the basal response of $\mathrm{B} 4 / 5$ to the CS prior to training. Then, we examined whether in vitro classical conditioning modified the ability of B4/5 to respond to the CS by counting the number of preparations in which $\mathrm{B} 4 / 5$ exhibited spike activity during the Post-Test greater than the basal response prior to training. In 7 of the 10 preparations trained with the paired protocol, B4/5 exhibited an increase in the total number of action potentials during the Post-Test as compared with the overall average measured during the Pre-Test. Similarly, in 7 of the 13 preparations trained with the unpaired protocol, B4/5 exhibited an increase in the total number of spikes in B4/5 during the Post-Test as compared with the overall average measured during the Pre-Test. Statistical analysis was performed on the $2 \times 2$ contingency table describ-

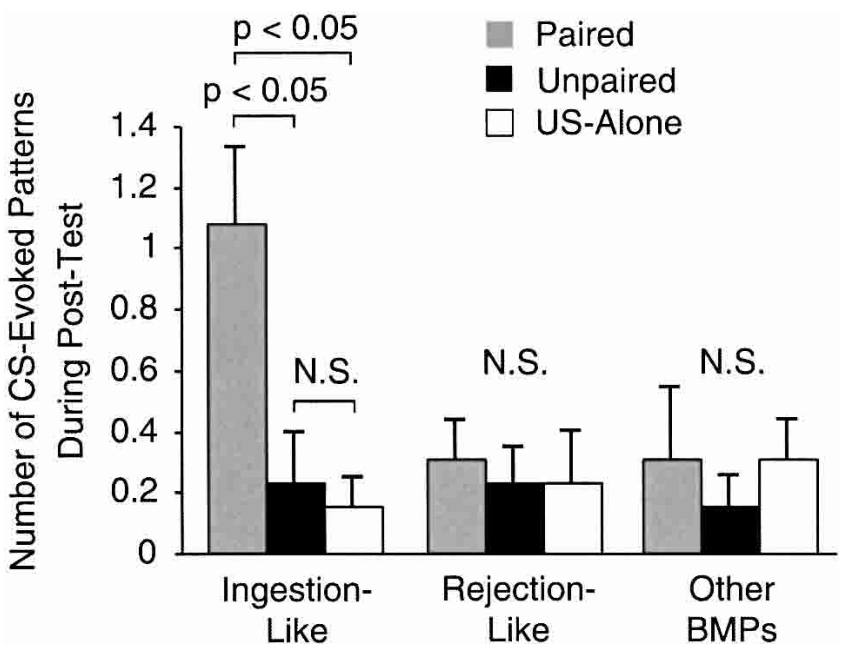

Figure 6 Paired training increased the number of ingestion-like BMPs evoked by the CS. The CS-evoked BMPs after training were classified using the criteria described in Figure 5. Patterns that did not fit either of the above criteria were designated "other BMPs." After paired training (gray), the CS elicited significantly more ingestion-like BMPs compared with unpaired (black) and US-alone (white) training. There was no difference in the number of rejection-like or other BMPs between paired, unpaired, and US-alone protocols. Thus, the increased number of CSevoked BMPs after training was almost entirely attributable to ingestionlike BMPs. 
A. Paired

1. Before Training

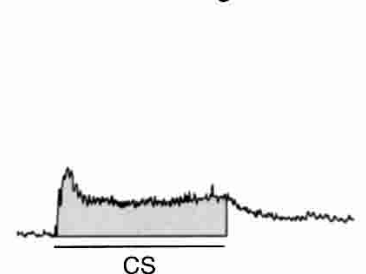

B. Unpaired

1. Before Training

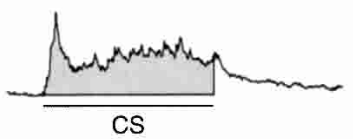

C.

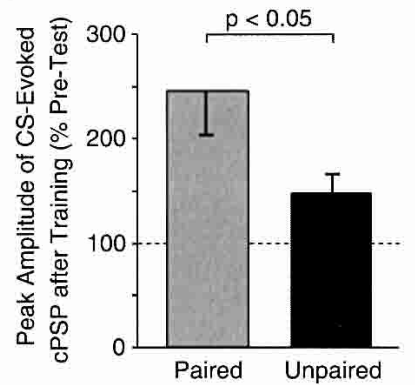

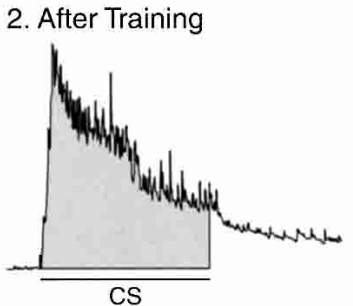

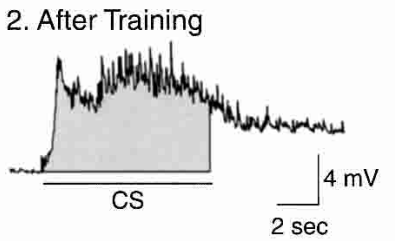

D.

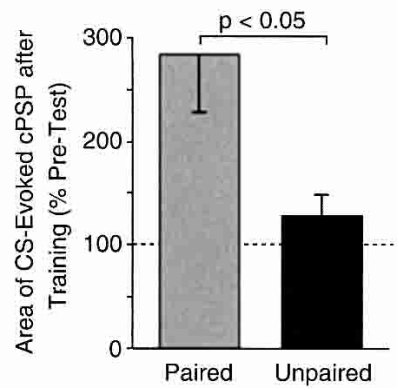

Figure 7 Classical conditioning produced an associative increase in the CS-evoked synaptic input to pattern initiating neuron B31/32. (A) Intracellular recording from B31/32 illustrating the complex postsynaptic potentials (CPSPS) evoked by the CS before $(A 1)$ and after $(A 2)$ paired training. (B) Recordings of CS-evoked CPSPs before (B1) and after (B2) unpaired training. In $A$ and $B$, the shaded area underneath each recording indicates the area over the 8-sec duration of the CS (see Materials and Methods). (C) The changes in the peak amplitude of the CS-evoked CPSP were measured after paired or unpaired training. Paired training induced a significantly greater increase in the amplitude of the CPSP as compared with unpaired training. $(D)$ The overall magnitude of the CS-evoked synaptic input was measured by integrating the CPSP over the 8-sec duration of the CS. Paired training induced a significantly greater increase in the area of the CPSP as compared with unpaired training. These results are consistent with a potentiation of the CS pathway as a result of classical conditioning.

ing the number of preparations, in which B4/5 exhibited CSevoked spike activity greater after training than the basal response measured prior to training. The Fisher exact test did not reveal any significant difference between the paired (7 of 10) and unpaired (7 of 13 ) groups ( $P=0.669$; Fig. $8 \mathrm{E}$ ).

Because in vitro classical conditioning failed to produce an associative change in the response of $\mathrm{B} 4 / 5$ to the CS, it was unlikely that any difference in synaptic input or intrinsic properties would be detected. Nevertheless, we examined these properties to compare the results of the in vitro analog with previous findings from in vivo classical conditioning (Lechner et al. 2000b). B4/5 exhibited a sustained train of action potentials in response to the CS. Thus, the 8-sec CS was not a practical stimulus to measure the synaptic input to $\mathrm{B} 4 / 5$. Therefore, a single $\mathrm{AT}_{4}$ stimulation $(0.5 \mathrm{msec})$ was used to evoke a cPSP in B4/5 before the Pre-Test and the Post-Test while the membrane potential of the neuron was current-clamped at $-80 \mathrm{mV}$. The average peak amplitude of the cPSP before the training did not differ between the paired and the unpaired groups (Fig. 8A1,B1). After training, the change in the peak amplitude of the cPSP in B4/5 in

the paired group $(139.3 \% \pm 13.1 \%$ of Pre-Test; $n=9)$ was not significantly different from that in the unpaired group $(126.7 \% \pm 20.4 \%$ of Pre-Test; $n=12 ; U=38, p=0.271$; Fig. $8 \mathrm{~A} 2, \mathrm{~B} 2, \mathrm{C})$. We also analyzed the net depolarization (i.e., the area of the cPSP) elicited by 0.5 -msec stimulation of $\mathrm{AT}_{4}$ over 400 msec. Before training, the average area of the cPSP did not differ between the paired and the unpaired groups (Fig. 8A1,B1). After training, the change in the area of the cPSP was not significantly different between the paired $(161.9 \% \pm 24.5 \%$ of Pre-Test; $n=9)$ and the unpaired $(145 \% \pm 22.4 \%$ of Pre-Test; $n=12)$ groups $(U=47, p=0.644$; Fig. 8A2,B2,D).

Finally, to examine whether classical conditioning induced changes in the membrane properties of $\mathrm{B} 4 / 5$, the resting membrane potential and input resistance of $\mathrm{B} 4 / 5$ were measured before and after training in paired- and unpaired-trained preparations. Before training, the average resting membrane potential did not differ between the paired $(-60.6 \pm 1.4 \mathrm{mV} ; n=9)$ and the unpaired $(-55.5 \pm 2.4 \mathrm{mV} ; n=12)$ groups $(U=31, p=0.11)$. After training, the change in the resting membrane potential of B4/5 in the paired and unpaired groups was not significantly different (Table 1). Before training, the average input resistance did not differ between the paired $(1.4 \pm 0.2 \mathrm{M} \Omega ; n=9)$ and the unpaired $(1.5 \pm 0.2 \mathrm{M} \Omega ; n=12)$ groups $(U=44.5, p=0.522)$. After training, the change in the input resistance of B4/5 in the paired and unpaired groups was not significantly different (Table 1 ).

These findings indicate that classical conditioning did not induce any pairing-specific changes in either the CS-evoked synaptic input to $\mathrm{B} 4 / 5$ or the intrinsic properties of the cell. Importantly, B4/5 also failed to manifest pairing-specific changes following behavioral conditioning (Lechner et al. 2000b). These results also indicate that the effects of paired training are expressed in some (e.g., B31/32), but not all (e.g., B4/5), elements of neural circuit for feeding. We next exploited the in vitro analog to identify the source(s) of the enhanced synaptic input (e.g., Fig. 7) to B31/32 during in vitro classical conditioning.

\section{Paired Training Did Not Produce an Associative Change in the Monosynaptic Connection From CBI-2 to $\mathrm{B} 31 / 32$}

Although a detailed description of the network mediating the CS is not complete, neurons such as CBI-2 represent potential sites for the associative plasticity underlying classical conditioning. CBI-2 is a command-like neuron that receives sensory input from the lips (Rosen et al. 1991; Mozzachiodi et al. 2000), makes monosynaptic connections with several CPG elements, including B31/32 (Rosen et al. 1991; Sánchez and Kirk 2000, 2001; Hurwitz et al. 2003), and has the ability to drive multiple BMPs (Rosen et al. 1991). Therefore, we explored the role of CBI-2 in classical conditioning.

A total of 29 preparations were trained in vitro with either paired $(n=14)$ or unpaired $(n=15)$ protocols. These preparations were used to explore the effects of in vitro classical conditioning on both the magnitude of the synapse from CBI-2 to B31/32 and the CS-evoked response in CBI-2 (see below).

Before training, the number of CS-evoked BMPs elicited during the Pre-Test did not differ significantly between the paired and the unpaired groups. As in previous experiments, paired training resulted in a significantly greater increase in the number of CS-evoked BMPs $(1.54 \pm 0.43$ BMPs; $n=14)$ than unpaired training $(0.57 \pm 0.23 \mathrm{BMPs} ; n=15) 60 \mathrm{~min}$ after conditioning $(U=58, p<0.05)$. First, the strength of the synaptic connection from CBI-2 to the ipsilateral B31/32 was tested before and after training. Because of the difficulty in maintaining simultaneous impalement of neurons CBI-2 and B31/32 throughout the duration of each experiment, it was possible to measure the magni- 
Table 1. Changes in Intrinsic Properties of Identified Neurons After in Vitro Classical Conditioning

\begin{tabular}{|c|c|c|c|c|c|c|}
\hline Neuron & $\begin{array}{l}\text { Preparations } \\
(n)\end{array}$ & $\begin{array}{l}\text { Intrinsic properties } \\
\text { (\% control) }\end{array}$ & $\begin{array}{l}\text { Paired } \\
\text { (P) }\end{array}$ & $\begin{array}{l}\text { Unpaired } \\
\text { (UP) }\end{array}$ & $U$ & $p$ \\
\hline B31/32 & $P=10 ; U P=10$ & Resting membrane potential & $\begin{array}{r}103.8 \% \pm 1.5 \\
868 \%+5.6\end{array}$ & $\begin{aligned} 101.6 \% & \pm 1.4 \\
83.6 \% & +5.1\end{aligned}$ & $\begin{array}{l}31 \\
41\end{array}$ & 0.162 \\
\hline B4/5 & $P=9 ; U P=12$ & $\begin{array}{l}\text { Resting membrane potential } \\
\text { Input resistance }\end{array}$ & $\begin{array}{l}94.8 \% \pm 3.1 \\
91.4 \% \pm 5.2\end{array}$ & $\begin{array}{l}101.7 \% \pm 4.1 \\
105.6 \% \pm 15.4\end{array}$ & $\begin{array}{l}38.5 \\
46\end{array}$ & $\begin{array}{l}0.286 \\
0.594\end{array}$ \\
\hline CBI-2 & $P=12 ; U P=13$ & $\begin{array}{l}\text { Resting membrane potential } \\
\text { Input resistance }\end{array}$ & $\begin{array}{l}95.6 \% \pm 4.6 \\
96.7 \% \pm 11.3\end{array}$ & $\begin{array}{l}97.6 \% \pm 3.3 \\
87.8 \% \pm 12.5\end{array}$ & $\begin{array}{l}68 \\
62\end{array}$ & $\begin{array}{l}0.605 \\
0.399\end{array}$ \\
\hline
\end{tabular}

Two of the intrinsic properties, resting membrane potential and input resistance, of buccal neurons B31/32, B4/5, and cerebral neuron CBI-2 were examined in reduced preparations before and after in vitro training with either paired (P) or unpaired (UP) protocols. The changes in resting membrane potential and input resistance after training were expressed as a percentage of those measured before training. The resting membrane potential and the input resistance of these neurons did not exhibit significant associative changes in response to classical conditioning (see $U$ and $p$ values from Mann-Whitney test).

tude of the synapse from CBI-2 to B31/32 in only 15 preparations (seven paired and eight unpaired) in the total group of 29 preparations trained in vitro (see Materials and Methods). Before training, the average peak amplitude of the PSP elicited in B31/32 by a $1-$ sec, $10-\mathrm{Hz}$ train in CBI-2 did not differ between the paired and the unpaired groups (Fig. 9A1,B1). After training, the change in the amplitude of the PSP was not significantly greater in the paired $(186.9 \% \pm 55.8 \%$ of Pre-Test; $n=7)$ than in the unpaired $(168.1 \% \pm 36.4 \%$ of Pre-Test; $n=8)$ groups $(U=28, p=1$; Fig. $9 \mathrm{~A} 2, \mathrm{~B} 2, \mathrm{C})$. The area of the PSP induced in B31/32 by CBI- 2 stimulation was also examined. Before training, the average area of the PSP in B31/32 did not differ between the paired and the unpaired groups (Fig. 9A1,B1). After training, the change in area of the PSP in $\mathrm{B} 31 / 32$ was not significantly greater in the paired $(169.4 \% \pm 51.4 \%$ of Pre-Test; $n=7)$ than in the unpaired $(182.1 \% \pm 74.2 \%$ of Pre-Test; $n=8)$ groups $(U=25, p=0.779$; Fig. 9A2,B2,D). Because the lack of any change in the magnitude of the synapse from CBI- 2 to B31/32 could be attributed to a failure of conditioning in those preparations in which the synapse was successfully measured, we also analyzed the difference in the number of CS-evoked BMPs in the 15 preparations used to study this synapse. The comparison of the difference in the number of CS-evoked BMPs between paired ( $1 \pm 0.33$ BMPs) and unpaired $(0 \pm 0.42$ BMPs) training did not achieve significance $(U=16.5, p=0.105)$ in the 15 preparations used to study the synapse from CBI-2 to B31/32. However, the dissimilarity of the $p$-values calculated for the difference in the number of CS-evoked BMPs $(p=0.105)$ and the change in the amplitude $(p=1.0)$ of the synapse from $\mathrm{CBI}-2$ to $\mathrm{B} 31 / 32$ indicated that the pairing-specific increase in the number of CS-evoked BMPs exhibited a trend toward significance after classical conditioning, whereas the amplitude of the synapse from CBI-2 to B31/32 was not changing in either paired or unpaired groups. These results indicate that an enhancement of the synaptic connection from CBI-2 to B31/32 was not the source of the pairing-specific increase in the CSevoked synaptic input to B31/32 observed after classical conditioning (Fig. 7).

\section{Paired Training Produced a Greater CS-Evoked Response in CBI-2 Than Unpaired Training}

The previous experiment indicated that the strength of the synapse from CBI-2 to B31/32 was not modified by classical conditioning. However, pairing-specific changes in the CS-evoked spike activity of CBI- 2 could, at least in part, contribute to increase the magnitude of the CS-evoked cPSP in B31/32. Therefore, the final experiment examined the effect of classical conditioning on the CS-evoked responses in CBI-2 (i.e., PSPs and spike activity) and on two intrinsic properties of CBI-2 (i.e., resting membrane potential and input resistance). The analysis was per- formed on the preparations used for the previous experiment in which paired training significantly increased the number of BMPs, compared with unpaired training.

First, we analyzed the effect of classical conditioning on the CS-evoked excitatory input to CBI-2. The stimulus used was shorter than the CS (i.e., $1 \mathrm{sec}$ vs. $8 \mathrm{sec}$ ), but it had the same frequency of stimulation $(5 \mathrm{~Hz}, 0.5-\mathrm{msec}$ pulses; see Materials and Methods) of the in vitro CS used during testing and training. We first analyzed the net depolarization (i.e., the area of the synaptic profile) elicited by $1-\mathrm{sec}, 5-\mathrm{Hz}$ stimulation of $\mathrm{AT}_{4}$ while the membrane potential of $\mathrm{CBI}-2$ was current-clamped at -80 $\mathrm{mV}$. Before training, the average area of the synaptic profile did not differ between the paired and the unpaired groups (Fig. $10 \mathrm{~A} 1, \mathrm{~B} 1)$. After training, the average area of the synaptic profile was greater in the paired $(309.7 \% \pm 89.6 \%$ of Pre-Test; $n=11)$ than in the unpaired(188.3\% $\pm 44.4 \%$ of Pre-Test; $n=13)$ groups, but the difference was not significant $(U=56, p=0.385$; Fig. 10A2,B2,D). However, it appeared that the first PSP in the train was enhanced greater than the other PSPs after paired training. Therefore, we examined whether the amplitude of the first PSP might be specifically facilitated. Before training, the average amplitude of the first $\mathrm{AT}_{4}$-evoked PSP in CBI-2 did not differ between the paired and the unpaired groups (Fig. 10A1,B1). The increase in the amplitude of the first PSP was significantly greater after paired $(254.9 \% \pm 51.1 \%$ of Pre-Test; $n=11)$ than after unpaired $(125.2 \% \pm 20.7 \%$ of Pre-Test; $n=13)$ training $(U=35$, $p<0.05$; Fig. 10A2,B2,C). To better understand the functional significance of the pairing-specific increase in the amplitude of the first PSP in CBI-2, we explored the occurrence of a possible correlation between such an increase and the difference in the number of CS-evoked BMPs. There was not a significant correlation between the change in the amplitude of the first CS-evoked PSP and the difference in the number of CS-evoked BMPs ( $r=0.35, p=0.09$, Spearman Rank Order Correlation), thus indicating that additional mechanisms are responsible for the increase in the number of CS-evoked BMPs induced by classical conditioning.

We next analyzed whether in vitro classical conditioning affected the ability of the CS pathway to activate CBI-2. Using a method similar to that used to analyze the spike activity of B4/5 in response to the CS, we calculated the basal response of CBI-2 prior to training and then the number of preparations, in which CBI-2 exhibited after training a response to the CS greater than the basal response. The total number of action potentials evoked in CBI-2 by four CSs during the Pre-Test did not differ between the paired and the unpaired groups $(U=69, p=0.641)$. Thus, these data were pooled and the overall average number of action potentials elicited in CBI-2 during the Pre-Test regardless of the type of training was $4.6 \pm 2.9(n=25)$. In 6 of the 12 prepara- 
A. Paired

\section{Before Training}

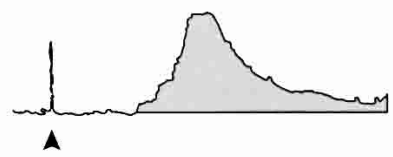

B. Unpaired

\section{Before Training}

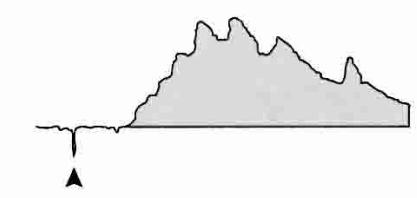

C.

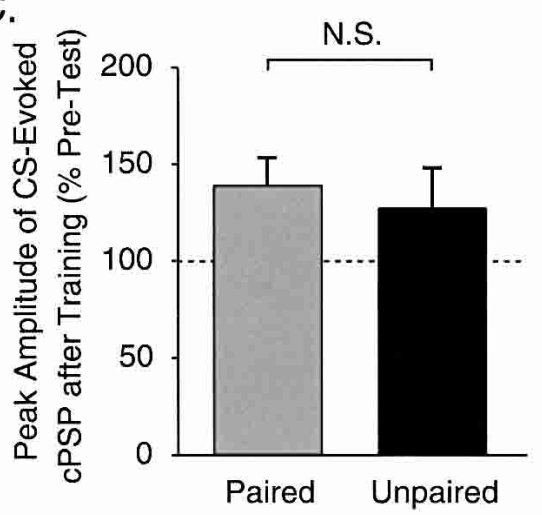

\section{After Training}
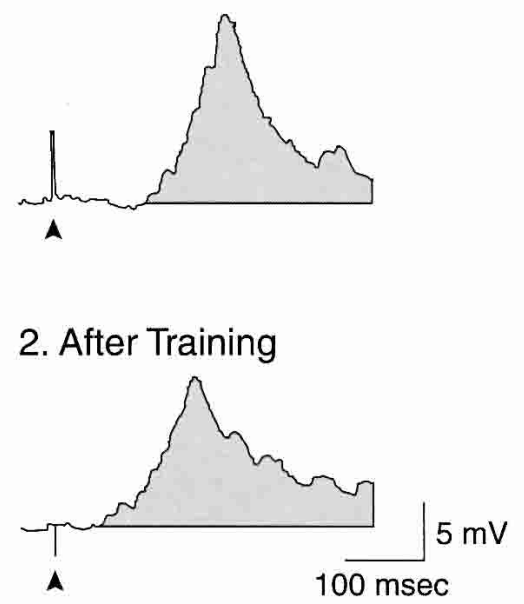

D.

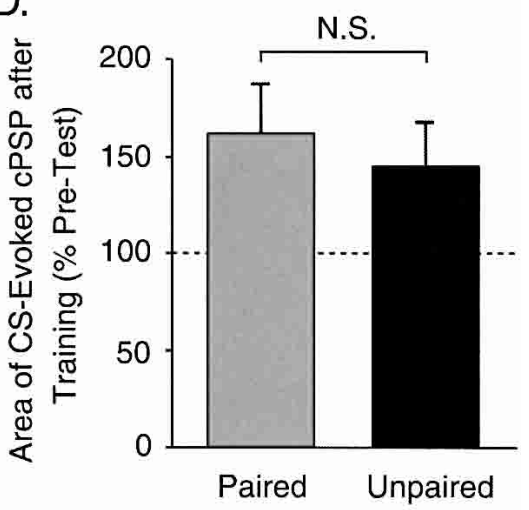

E.

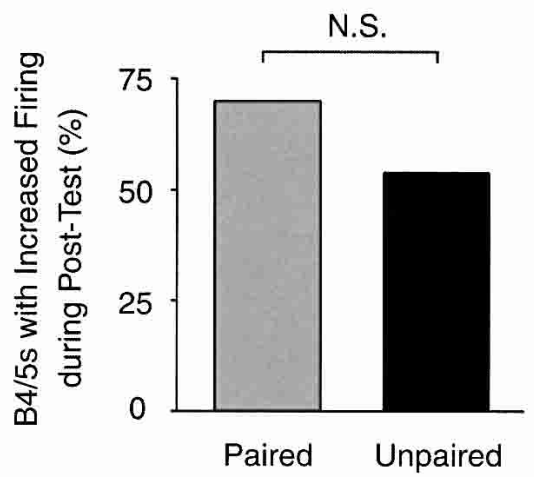

Figure 8 Classical conditioning did not produce associative changes in the CS-evoked synaptic input to B4/5. (A) Intracellular recording from B4/5 illustrating the CPSPs evoked by $0.5-\mathrm{msec}$ stimulation of $\mathrm{AT}_{4}$ before $(A 1)$ and after $(A 2)$ paired training. (B) Recordings of CPSPs in B4/5 before (B1) and after (B2) unpaired training. In $A$ and $B$, the arrowhead below each recording indicates the artifact of $\mathrm{AT}_{4}$ electrical stimulation, and the shaded area underneath each trace indicates the area over 400 msec used to measure the amount of depolarization in $\mathrm{B} 4 / 5$ produced by $\mathrm{AT}_{4}$ stimulation. (C) Change in the peak depolarizing amplitude of the CPSP in B4/5 after paired or unpaired training. No significant pairing-specific change in the CPSP peak amplitude was detected. (D) Change in the area of the synaptic input to B4/5 produced by paired or unpaired training. No significant pairing-specific change in the CPSP area was detected. (E) Percentage of preparations that exhibited an increased firing activity in B4/5 after training. The analysis of the contingency table (Fisher exact test: paired vs. unpaired) did not reveal any significant difference.

tions trained with the paired protocol, CBI-2 exhibited an increase in the total number of action potentials during the Post-Test as compared with the overall average measured during the Pre-Test (Fig. $11 \mathrm{~A} 1, \mathrm{~A} 2)$. In contrast, none of the 13 preparations trained with the unpaired protocol exhibited an increase in the total number of spikes in CBI-2 during the Post-Test as compared with the overall average measured during the Pre-Test (Fig. 11B1,B2). Statistical analysis was performed on the $2 \times 2$ contingency table describing the number of preparations, in which CBI-2 exhibited CSevoked spike activity greater after training than the basal response measured before training. The Fisher exact test indicated a significant difference between the paired (6 of 12) and unpaired (0 of 13) groups $(P<0.05$; Fig. 11C). These results indicate that classical conditioning enhances the CS-evoked input to CBI-2, which leads to increased CS-evoked spike activity in CBI-2. In addition, a significant positive correlation was detected between the change in the number of CS-evoked action potentials in CBI-2 and the difference in the number of CS-evoked BMPs $(r=0.43, p<0.05$, Spearman Rank Order Correlation). The pairing-specific increase in the spike activity in CBI-2 may, in turn, contribute, at least in part, to the associative enhancement of the CS-evoked synaptic input to B31/32 (e.g., Fig. 7). However, we cannot rule out the possibility that components of the enhanced CS-evoked input to B31/32 were produced by synaptic contributions from other command-like neurons such as CBI-1, CBI-3, or CBI-12, which converge onto B31/32 (Rosen et al. 1991; Sánchez and Kirk 2002).

Finally, to examine whether classical conditioning induced changes in the membrane properties of CBI-2, the resting membrane potential and input resistance of CBI-2 were measured before and after training in paired- and unpaired-trained preparations. Before training, the average resting membrane potential of CBI-2 did not differ between the paired $(-65.6 \pm 1.8 \mathrm{mV}$; $n=12)$ and the unpaired $(-67.8 \pm 1.5 \mathrm{mV}$; $n=13)$ groups $(U=63.5, p=0.446)$. After training, the change in the resting membrane potential of CBI-2 in the paired and unpaired groups was not significantly different (Table 1). Before training, the average input resistance did not differ between the paired $(6.2 \pm 0.8 \mathrm{M} \Omega ; n=12)$ and the unpaired $(6.8 \pm 0.7 \mathrm{M} \Omega ; n=13)$ groups $(U=70.5, p=0.703)$. After training, the change in the input resistance of CBI-2 in the paired and unpaired groups was not significantly different (Table 1). Therefore, in vitro classical conditioning did not affect either the resting membrane potential or the input resistance of CBI-2. 


\section{A. Paired}

\section{Before Training}

B31/32 maranchrshor

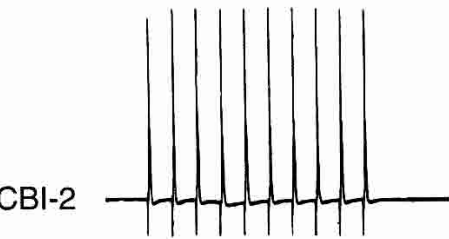

\section{B. Unpaired}

\section{Before Training}

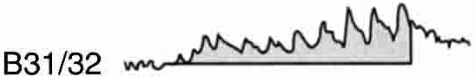

$\mathrm{CBI}-2$

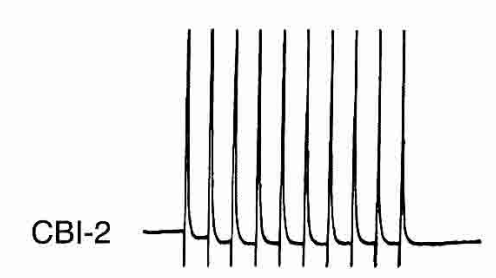

\section{C.}

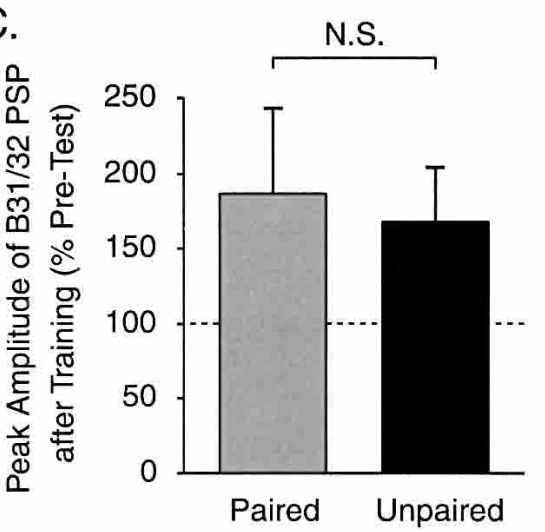

\section{After Training}
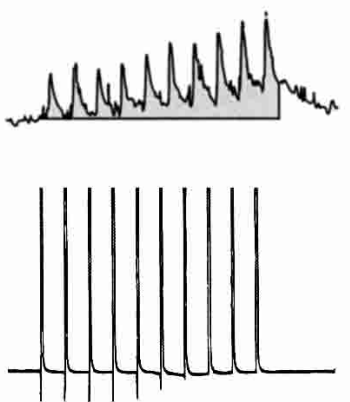

\section{After Training}
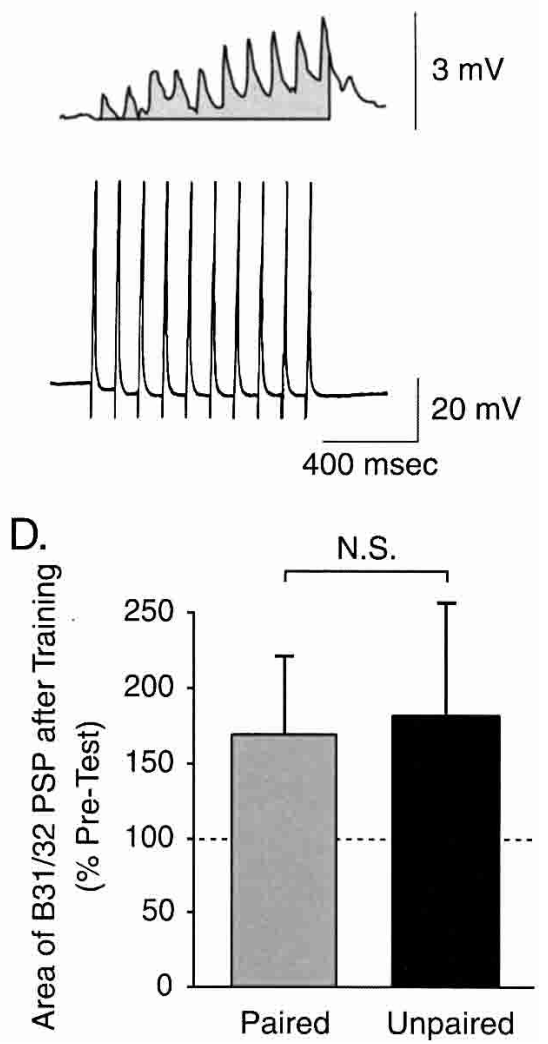

Figure 9 Classical conditioning did not produce an associative change in the magnitude of the synaptic connection from CBI-2 to B31/32. (A) Simultaneous intracellular recordings of CBI-2 (lower trace) and $\mathrm{B} 31 / 32$ (upper trace) before $(A 1)$ and after $(A 2)$ paired training. (B) Recordings of $\mathrm{CBI}-2$ and $B 31 / 32$ before $(B 1)$ and after (B2) unpaired training. The synaptic response in $B 31 / 32$ was probed with a train of 10 spikes $(1 \mathrm{sec}, 10 \mathrm{~Hz})$ in $\mathrm{CBI}-2$ while the membrane potential of $\mathrm{B} 31 / 32$ was current-clamped at $-80 \mathrm{mV}$. In $A$ and $B$, the shaded area underneath each recording from $B 31 / 32$ indicates the area over a 1-sec duration. (C) Change in the peak amplitude of the synaptic connection from $\mathrm{CBI}-2$ to $\mathrm{B} 31 / 32$ after paired or unpaired training. No significant pairing-specific change in the peak amplitude was detected. $(D)$ Change in the area of the synaptic profile of the connection from $\mathrm{CBI}-2$ to $\mathrm{B} 31 / 32$ produced by paired or unpaired training. No significant pairingspecific change in the area was detected.

\section{DISCUSSION}

The present study describes the development and analysis of an in vitro analog of classical conditioning of feeding behavior in Aplysia. The reduced preparation, which consisted of the cerebral and buccal ganglia, was classically conditioned by pairing stimulation of peripheral nerves, $\mathrm{AT}_{4}$ and $\mathrm{En}_{2}$, used to mimic the CS and the US, respectively (Fig. 1A). Several lines of evidence from previous studies support the suitability of using $\mathrm{AT}_{4}$ and $\mathrm{En}_{2}$ as analogs of the CS and US (see Materials and Methods for details). In addition, we demonstrated that electrical stimulation of $\mathrm{AT}_{4}$ (i.e., the analog of the CS) induced spike activity in sensory neurons in the cerebral ganglion (Fig. 2), which were very likely mechanoafferents (Rosen et al. 1979, 1982). Paired stimulation of $\mathrm{AT}_{4}$ and $\mathrm{En}_{2}$ resulted in a greater probability of CS-evoked ingestion-like BMPs occurring $60 \mathrm{~min}$ after training (Figs. 3A, 4A, 6). Moreover, paired training increased the CS-evoked depolarization of neuron B31/32 (Fig. 7), which is thought to play a key role in initiating the protraction phase of consummatory feeding behavior (Susswein and Byrne 1988; Hurwitz et al. 1994, 1996; Susswein et al. 2002). However, classical conditioning did not significantly change either the synaptic input to multifunctional neuron B4/5 (Fig. 8) or its intrinsic membrane properties (Table 1), thus indicating that B4/5 is not a locus of plasticity that contributes to classical conditioning.

The changes in activity of the feeding CPG and synaptic input to B31/32 were specifically related to paired training (Figs. $3 \mathrm{~A}, 4 \mathrm{~A}, 6,7)$. Moreover, in the absence of stimulation of the CS pathway, there was no significant change in the spontaneous activity of the CPG (Fig. 4B), indicating that the changes induced by the in vitro analog of classical conditioning were expressed selectively in response to activity in the CS pathway. Importantly, the changes in the CS-evoked fictive feeding and the synaptic input to B31/32, which were induced by the in vitro analog of classical conditioning, were virtually identical to those produced by behavioral training (Lechner et al. 2000b). Thus, the neural changes produced by behavioral conditioning can be reproduced in vitro by training the isolated nervous system with paired presentation of the CS and US. This validation of the in vitro analog lays the foundation for future analyses of the cellular and molecular mechanisms underlying classical conditioning.

\section{Sites of Associative Plasticity in the In Vitro Analog of Classical Conditioning}

The enhancement of the CS-evoked synaptic input to B31/32 produced by in vitro classical conditioning was not accompanied by pairing-specific changes in its resting membrane potential or input resistance (Table 1). A possible explanation for this finding is that $\mathrm{B} 31 / 32$ is not the site of the associative plasticity produced 
In Vitro Analog of Classical Conditioning

\section{A. Paired}

\section{Before Training}

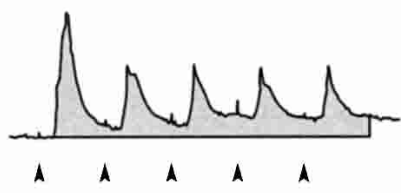

\section{B. Unpaired}

\section{Before Training}

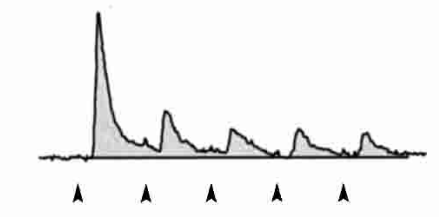

C.

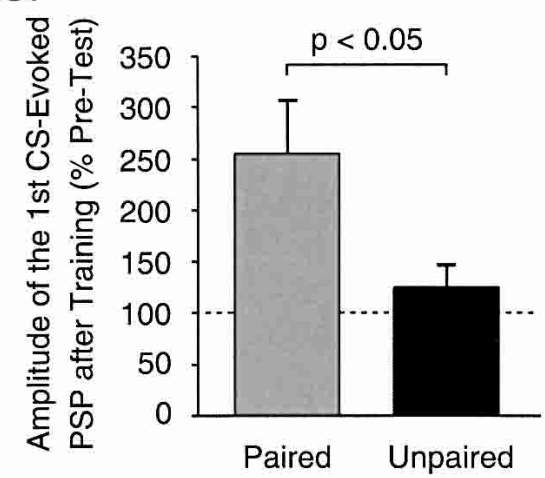

2. After Training

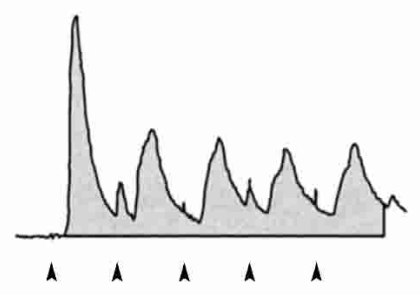

2. After Training

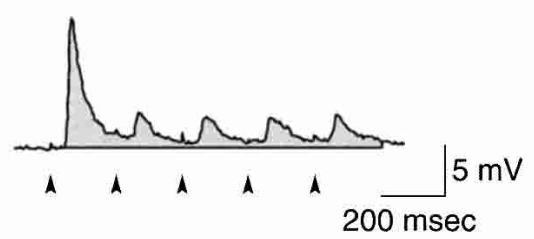

D.

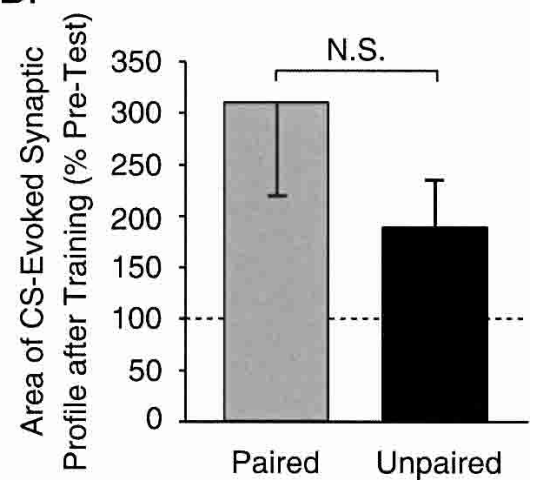

Figure 10 Classical conditioning induced an associative increase in the magnitude of the initial CS-evoked synaptic response of CBI-2. (A) Intracellular recording illustrating the PSPs in CBI-2 that were elicited by $\mathrm{AT}_{4}$ stimulation before $(A 1)$ and after $(A 2)$ paired training. $(B)$ Intracellular recording illustrating the PSPs in $\mathrm{CBI}-2$ that were elicited by $\mathrm{AT}_{4}$ stimulation before $(B 1)$ and after (B2) unpaired training. The stimulus used in $A$ and $B$ consisted of five brief $A_{4}$ shocks $(0.5 \mathrm{msec})$ at 5 $\mathrm{Hz}$, which elicited five PSPs in CBI-2. In $A$ and $B$, the artifact of each $\mathrm{AT}_{4}$ stimulation is indicated with an arrowhead below the recordings. Also, the shaded area underneath each recording indicates the area of the five PSPs over a 1 -sec duration (i.e., synaptic profile). (C) Paired training induced a significant increase in the amplitude of the first PSP in CBI-2, as compared with unpaired training. $(D)$ The area of the synaptic profile of $\mathrm{CBI}-2$ response to $\mathrm{AT}_{4}$ stimulation appeared to increase after training, but the effect was not statistically significant. (E) Percentage of preparations that exhibited an increased firing activity in $\mathrm{CBI}-2$ after training. The analysis of the contingency table (Fisher exact test: paired vs. unpaired) revealed that the difference was statistically significant.

by in vitro paired training and that the locus of plasticity is located upstream of B31/32, along the CS pathway.

Therefore, the next steps of our analysis focused on identifying sites of plasticity that could contribute to the increased CS-evoked input to B31/32. Although the CS pathway has not been entirely characterized, some elements have been described and are putative candidates for learning-related plasticity. For example, the cerebral command-like interneuron CBI-2 receives mechanosensory inputs from the lips via $\mathrm{AT}_{4}$ (Rosen et al. 1991; Mozzachiodi et al. 2000). Also, CBI-2 can drive patterned activity in the feeding CPG (Rosen et al. 1991; Church and Lloyd 1994; Jing and Weiss 2001, 2002) via monosynaptic connections with CPG elements, including B31/32 (Rosen et al. 1991; Sánchez and Kirk 2000, 2001, 2002; Hurwitz et al. 2003). Although training appeared to affect the strength of the synapse from CBI-2 to
B31/32 in a nonassociative manner, our results indicate that this synaptic connection was not specifically affected by paired training (Fig. 9). Because the synapse from CBI-2 to $\mathrm{B} 31 / 32$ was probed with a constant number of action potentials in CBI-2, the lack of pairing-specific changes at this synaptic site did not exclude a possible role of CBI- 2 in the pairing-specific plasticity accompanying classical conditioning. For example, an increase in CS-evoked spike activity in CBI-2 could lead to an enhanced synaptic input to B31/32. Therefore, the search for the loci of associative plasticity focused on pairing-specific changes in the intrinsic properties of CBI-2 as well as in the $\mathrm{AT}_{4}$-evoked excitatory input to CBI-2. The resting membrane potential and the input resistance of CBI-2 were not affected by classical conditioning (Table 1). However, the response of CBI-2 to $\mathrm{AT}_{4}$ stimulation was enhanced following paired training. Specifically, the CS-evoked spike discharge and synaptic input to CBI-2 were increased after paired training (Figs. 10, 11). Classical conditioning appeared to enhance the amplitude of the first CSevoked PSP in CBI-2. Because the initial triggering stimulus is often the most important to elicit a fixed action pattern (Getting 1975; Staras et al. 1999), one might expect that an increase in the initial synaptic input to CBI-2 would play a key role in producing the conditioned response. However, the lack of a significant correlation between the increase in the amplitude of the first PSP in CBI-2 and the difference in the number of CS-evoked BMPs may indicate that the pairing-specific enhancement of the amplitude of the first CS-evoked PSP was not sufficient to account for the increase in the number of CS-evoked BMPs induced by classical conditioning. This result indicates the involvement of additional mechanisms in the formation of appetitive associative memory. Interestingly, a significant positive correlation was detected between the change in the number of CS-evoked action potentials in CBI-2 and the difference in the number of CS-evoked BMPs, which implies that an enhancement of the sensory input from the lips may have a functional relevance in the increase in fictive feeding following classical conditioning. To resolve this issue, further experiments that focus on the properties of the cerebral sensory neurons are required. Associative changes in the properties of commandlike neurons in molluscs have been previously reported by Davis and Gillette (1978) and by Kemenes et al. (2002). In Pleurobranchea, food stimuli, which normally excite command neurons in naive animals, inhibit these neurons when animals are trained with an avoidance conditioning paradigm (Davis and Gillette 1978). In addition, appetitive conditioning in Limnaea produces an associative increase in the CS-evoked spike activity in sensory neurons (Staras et al. 1999) and in the CS-evoked depolarization of the modulatory neuron CV1a (Kemenes et al. 2002), which is homologous to CBI-2 in Aplysia (Kemenes et al. 2001). 
A. Paired 1. Pre-Test

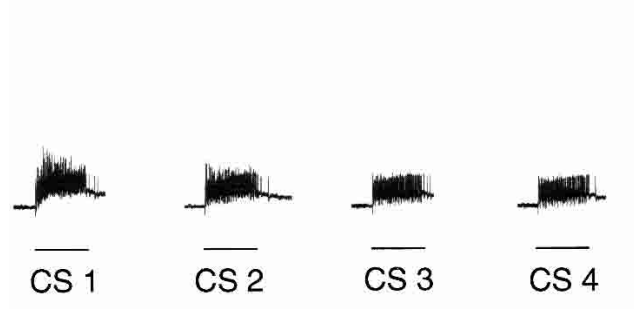

B. Unpaired

1. Pre-Test
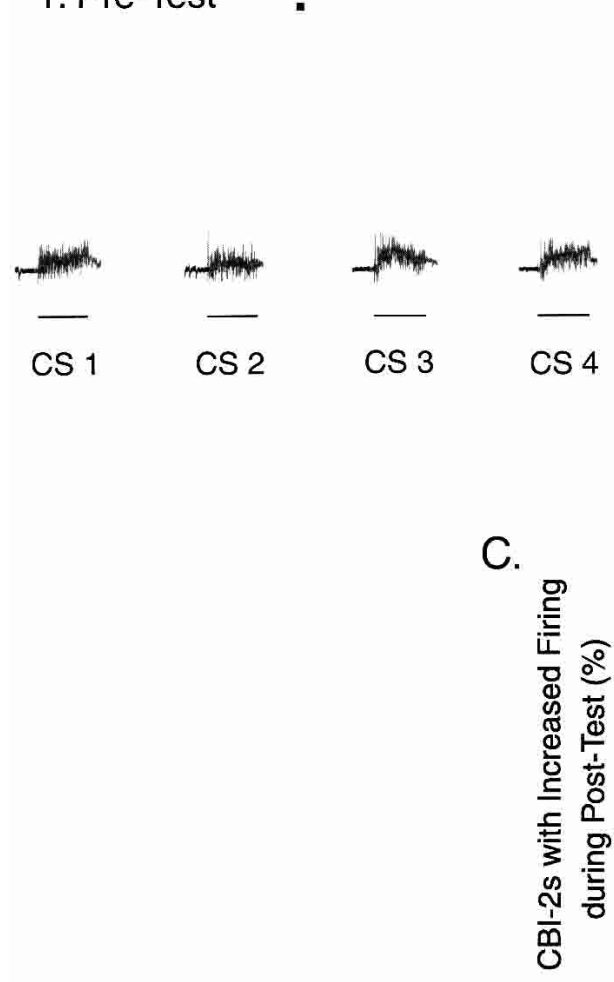

\section{Post-Test}

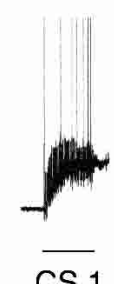

CS 1

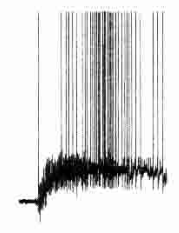

cs 2

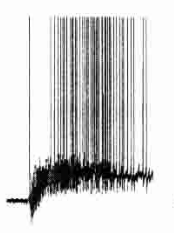

CS 3

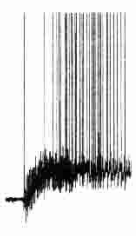

CS 4

2. Post-Test

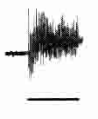

CS 1

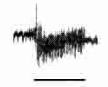

CS 2

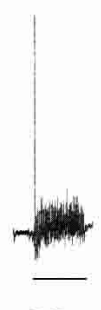

CS 3

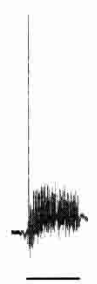

CS 4

$\underset{8 \mathrm{sec}}{\mathrm{f}} 10 \mathrm{mV}$

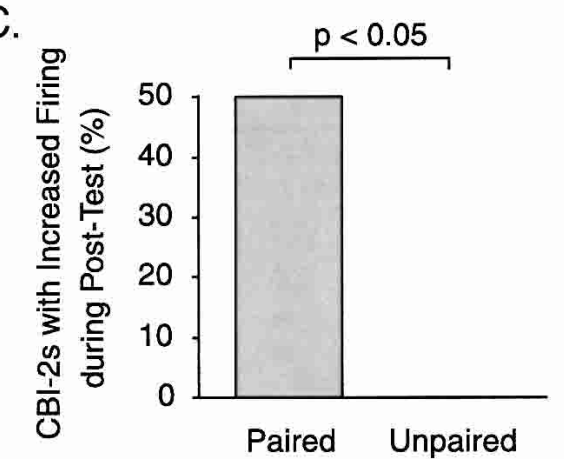

Figure 11 Classical conditioning induced an associative increase in the spike activity of CBI-2 in response to the CS. (A) Intracellular recording illustrating the spike activity in CBI-2 that was elicited by four CSs during the Pre-Test (A1) and the Post-Test (A2) in a preparation trained with the paired protocol. (B) Intracellular recording illustrating the spike activity in CBI-2 that was elicited by four CSs during the Pre-Test (B1) and the Post-Test (B2) in a preparation trained with the unpaired protocol. (C) Percentage of preparations that exhibited an increased firing activity in CBI- 2 after training. The analysis of the contingency table (Fisher exact test: paired vs. unpaired) revealed that the difference was statistically significant.

\section{Are Cerebral Sensory Neurons Sites of Associative Plasticity Underlying Classical Conditioning of Feeding?}

The results discussed above indicate that some aspects of associative plasticity may be occurring upstream of CBI-2, presumably at the level of the sensory neurons. The cerebral ganglion contains sensory neurons in the $\mathrm{J}$ and $\mathrm{K}$ clusters (Rosen et al. 1979), which contribute to a variety of behaviors, including feeding (Rosen et al. 1979, 1982, 1989). The sensory neurons in the J and $\mathrm{K}$ clusters encode mechanical inputs from different regions of the head, including the lips (Rosen et al. 1979, 1982, 1989). In addition, the present study demonstrated that electrical stimulation of $\mathrm{AT}_{4}$ (i.e., the analog of the CS), in the intensity range used for testing and training procedures, activates sensory neurons in the $\mathrm{J}$ and $\mathrm{K}$ clusters (Fig. 2).
In Aplysia, classical conditioning analogs and classical conditioning of withdrawal reflexes produce pairing-specific modifications occurring at the level of sensory neurons (Hawkins et al. 1983; Walters and Byrne 1983; Murphy and Glanzman 1997; Antonov et al. 2001, 2003). Therefore, our findings imply the intriguing hypothesis that in Aplysia, mechanisms of associative plasticity that have been identified in sensory neurons that mediate defensive behaviors may play a role also in appetitive forms of classical conditioning. If this hypothesis is correct, the Aplysia nervous system would use analogous strategies (i.e., plasticity occurring at the level of the sensory neurons) to express associative memory of different behaviors, regardless of either the nature of the behavior (appetitive or defensive) or the complexity of its underlying neural network (e.g., simple reflexes vs. complex 
appetitive behaviors). Future experiments will be designed to test the involvement of cerebral sensory neurons in classical conditioning of feeding behavior.

It is important to note that the present study does not exclude the possibility that additional sites of associative plasticity would contribute to classical conditioning of feeding behavior. For example, CBI-2 is only one of the elements of a network of command-like interneurons that includes CBI-1, CBI-3, and CBI12 (Rosen et al. 1991; Hurwitz et al. 1999; Jing and Weiss 2001). These neurons process sensory information and control feeding through synaptic connections with CPG elements, including B31/32 (Hurwitz et al. 1997, 1999; Sánchez and Kirk 2000, 2001, 2002; Morgan et al. 2001). One intriguing locus for pairing-specific plasticity is also neuron B51 (Plummer and Kirk 1990), which has been implicated in operant conditioning of feeding behavior both in vivo (Brembs et al. 2002) and in vitro (Nargeot et al. 1999a,b). Therefore, future analyses will explore additional elements as potential loci of plasticity associated with classical conditioning.

\section{In Vitro Classical Conditioning in Other Invertebrate Model Systems}

In the past 30 years, several in vitro analogs of classical conditioning have been developed using invertebrate model systems (for review, see Sahley and Crow 1998). Most of the examples include responses to aversive stimuli in Aplysia, Drosophila, Hermissenda, Helix, Pleurobranchaea, and in the leech (e.g., Crow and Alkon 1978; Lukowiak and Sahley 1981; Hawkins et al. 1983; Walters and Byrne 1983; Maksimova and Balaban 1984; Kovac et al. 1986; Buonomano and Byrne 1990; Sahley et al. 1994; Sahley 1995; Talk and Matzel 1996; Murphy and Glanzman 1997; Bao et al. 1998; Gandhi and Matzel 2000; Antonov et al. 2001, 2003; Balaban et al. 2001; Dubnau and Tully 2001). These studies provided important contributions to the knowledge of the biophysical processes and the signal transduction cascades, which participate in the formation and retention of aversive forms of associative memory. Despite the development of these preparations, however, little information is available about the mechanisms of classical conditioning of appetitive forms of behavior (Sahley et al. 1990; Menzel and Müller 1996; Kemenes et al. 1997; Benjamin et al. 2000). Kemenes et al. (1997) recently developed a reduced preparation of the nervous system of Lymnaea that is amenable to the study of classical conditioning of feeding behavior. The semiintact preparation in Lymnaea exhibits associative changes in the feeding CPG very similar to those expressed by the Aplysia reduced preparation, such as pairing-specific increase in the CSevoked fictive feeding (Kemenes et al. 1997).

\section{MATERIALS AND METHODS}

\section{General Methods}

Aplysia californica (80-350 g) were obtained from Alacrity Marine Biological Specimens and Marinus and housed individually in perforated plastic cages, floating in aerated seawater tanks at $15^{\circ} \mathrm{C}$. Animals were fed $\sim 1 \mathrm{~g}$ of dried seaweed three times a week.

\section{Dissection}

Prior to dissection, the motivational state of an animal was tested by presenting it with a piece of seaweed. If the animal began to eat, it was anesthetized by an injection of isotonic $\mathrm{MgCl}_{2}$ equivalent to $50 \%$ of its body mass. Animals that did not eat within 5 min were not used for the study. An incision was made along the midline of the ventral foot to expose the buccal mass and the esophagus. The most medial-ventral branch (designated branch 4 ) of the right anterior tentacle nerve (AT; for nomenclature, see Jahan-Parwar and Fredman 1976), which terminates in the lip region of the animal, was retained (Fig. 1A). All other peripheral nerves of the cerebral ganglion were cut short. The esophagus and the buccal mass together with the cerebral and buccal ganglia were removed and transferred to a chamber containing artificial seawater with a high concentration of divalent cations (high divalent ASW) composed of $210 \mathrm{mM} \mathrm{NaCl}, 10 \mathrm{mM} \mathrm{KCl}$, $145 \mathrm{mM} \mathrm{MgCl}_{2}, 20 \mathrm{mM} \mathrm{MgSO}$, $33 \mathrm{mM} \mathrm{CaCl}_{2}$, and $10 \mathrm{mM}$ HEPES ( $\mathrm{pH}$ adjusted to 7.5 with $\mathrm{NaOH}$ ). The high divalent ASW was used to decrease neural activity during further dissection (Byrne et al. 1978). Selected peripheral nerves of the right buccal ganglion were retained for extracellular recording and stimulation (Fig. 1A). The cerebral and the buccal ganglia were then pinned to the bottom of a Petri dish coated with silicone elastomer (Sylgard, Dow Corning). The buccal ganglia were pinned with the caudal surface up, which allowed access to neurons B31/ 32 and $\mathrm{B} 4 / 5$. The cerebral ganglion was pinned with the ventral surface up, which allowed access to neuron CBI-2 and to the sensory neurons in the $\mathrm{J}$ and $\mathrm{K}$ clusters. In the first series of experiments, the connective tissue sheath that covers the ganglia was left intact. In Experiments 2-5, the sheath was removed from the caudal surface of the right buccal ganglion to allow for intracellular recordings from neurons B31/32 or B4/5 (see below). In Experiments 4 and 5, the right $\mathrm{M}$ cluster (Ono and McCaman 1980; Rosen et al. 1991) of the cerebral ganglion was desheathed to expose the soma of command-like interneuron CBI-2. The temperature of the static bath was maintained at $15^{\circ} \mathrm{C}$ with a feedback-controlled peltier cooling device (Model SE 5010; Marlow Industries). The high divalent ASW was exchanged for normal ASW for $10 \mathrm{~min}$ prior to the beginning of an experiment, once the extracellular electrodes for both stimulation and recording were in place (see below). The normal ASW was composed of $450 \mathrm{mM} \mathrm{NaCl}, 10 \mathrm{mM} \mathrm{KCl}, 30 \mathrm{mM} \mathrm{MgCl}_{2}, 20 \mathrm{mM} \mathrm{MgSO}_{4}, 10$ $\mathrm{mM} \mathrm{CaCl}_{2}$, and $10 \mathrm{mM}$ HEPES (pH adjusted to 7.5 with $\mathrm{NaOH}$ ).

\section{Extracellular Nerve Recordings}

Previous in vivo recordings indicate that trains of large-unit activity in nerves $\mathrm{I}_{2} \mathrm{n}, \mathrm{Rn}_{1}$, and $\mathrm{Bn}_{2,1}$ are associated with the protraction, closure, and retraction, respectively, of the radula/ odontophore during feeding (Morton and Chiel 1993a; Hurwitz et al. 1996). Moreover, in vitro recordings indicate that BMPs, which represent fictive feeding, can be recorded from $\mathrm{I}_{2} \mathrm{n}, \mathrm{Rn}_{1}$, and $\mathrm{Bn}_{2,1}$ (e.g., Morton and Chiel 1993b; Nargeot et al. 1997; Kabotyanski et al. 2000; Lechner et al. 2000b). Thus, in this in vitro analog of classical conditioning, fictive feeding (i.e., BMPs) was monitored by placing silver electrodes on nerves $I_{2} n, R_{1}$, and $\mathrm{Bn}_{2,1}$ (Nargeot et al. 1997) of the right buccal ganglion (Figs. $1 \mathrm{~A}, 3,5$; see below). All extracellular electrodes were isolated from the surrounding bath using petroleum jelly (Vaseline; Sherwood Medical). Signals were amplified with a differential AC amplifier (Model 1700; A-M Systems), displayed on a chart recorder (Model TA11; Gould Instrument System Inc.) and stored on magnetic tape (Model 3000; Vetter).

\section{Extracellular Nerve Stimulation}

In this in vitro analog of classical conditioning, electrical stimulation (4 sec, $10 \mathrm{~Hz}, 0.5$-msec pulses) of the right $\mathrm{En}_{2}$, which innervates the buccal mass (Schwarz and Susswein 1986), was selected to mimic the US used in intact animals. The duration and frequency of the stimulus were similar to trains of activity recorded in vivo from $\mathrm{En}_{2}$ during feeding (Brembs et al. 2002). $\mathrm{En}_{2}$ mediates several aspects of feeding behavior such as conveying efferent activity that controls peristaltic movements of the gut (Lloyd et al. 1988) and conveying afferent activity that encodes information related to feeding arousal (Susswein et al. 1984) and satiety (Kuslansky et al. 1978, 1987). In addition, in vitro, electrical stimulation of $\mathrm{En}_{2}$ evokes an excitatory synaptic input in several CPG elements, including B31/32 and B4/5 (Susswein and Byrne 1988; Nargeot et al. 1997, 1999c) and elicits BMPs. Several lines of evidence indicate that $\mathrm{En}_{2}$ also mediates key aspects of the reinforcement during appetitive associative learning. First, a train of activity occurs in En $_{2}$ every time a bolus of food is ingested, whereas $\mathrm{En}_{2}$ is relatively silent in the absence 
of food in the foregut (Brembs et al. 2002). This observation indicates that $\mathrm{En}_{2}$ carries sensory information (mechanical and/ or chemical) about the presence of food in the buccal cavity. Second, presentation of food to the lips reliably elicits a bite, but this stimulus was not sufficient to induce in vivo classical conditioning. In fact, only if the food was ingested did associative learning occur (Lechner et al. 2000a). Third, bilateral lesions of $\mathrm{En}_{2}$ impair in vivo classical conditioning without affecting feeding behavior (Lechner et al. 2000a). These results indicate that afferent pathways originating in the epithelia of the buccal mass and/or foregut and conveyed by $\mathrm{En}_{2}$ contribute to appetitive reinforcement during associative learning of feeding behavior, whereas US-mediating pathways originating in the lips do not seem to play a role in appetitive reinforcement. Fourth, stimulation of $\mathrm{En}_{2}$ serves as a reinforcer to modify behavior and neural activity in a training paradigm used for operant conditioning of Aplysia feeding behavior both in vivo (Brembs et al. 2002) and in vitro (Nargeot et al. 1997). Finally, $\mathrm{En}_{2}$ is necessary in an operant paradigm for learning that food is inedible (Susswein and Schwarz 1983; Schwarz and Susswein 1986). Thus, En ${ }_{2}$ appears to be a reinforcement pathway that contributes to both classical and operant conditioning.

In this in vitro analog of classical conditioning, electrical stimulation of $\mathrm{AT}_{4}$ ( $8 \mathrm{sec}, 5 \mathrm{~Hz}, 0.5-\mathrm{msec}$ pulses) was selected to mimic the CS that was used in intact animals (Lechner et al. $2000 \mathrm{a}, \mathrm{b})$. The frequency of $\mathrm{AT}_{4}$ stimulation used in the present study was similar to that recorded in vivo during mechanical stimulation of the tentacles (Anderson 1967; Fredman and Jahan-Parwar 1980). The AT nerve mediates several aspects of feeding behavior. For example, AT conveys afferent activity that encodes information about both mechanical and chemical stimuli (Anderson 1967; Rosen et al 1979, 1982; Xin et al. 1995) that signal the presence of food on the lips. In addition, AT conveys efferent activity that controls the movement of the lips (Perrins and Weiss 1996). Several lines of evidence indicate that $\mathrm{AT}_{4}$ also mediates aspects of the tactile CS that was used for in vivo classical conditioning. First, mechanical stimulation of the lips activates sensory neurons whose somata are located in the lateral (J) and medial (K) mechanoafferent clusters in the cerebral ganglion (Rosen et al. 1979) and whose axons project in cerebral nerves including $\mathrm{AT}_{4}$ (Rosen et al. 1979, 1982). Second, cerebral mechanoafferent neurons in the $\mathrm{J}$ and $\mathrm{K}$ clusters exhibit antidromic action potentials in response to brief electrical stimulation of $\mathrm{AT}_{4}$ $(0.5 \mathrm{msec}$ ) with an intensity $>4.2 \mathrm{~V}$ (Lechner et al. 2000b). These results indicate that the in vitro CS activates the mechanosensory pathways from the lips, which presumably were activated in vivo by the tactile CS. Finally, Lechner et al. (2000b) found that in vivo classical conditioning (1) increased the probability that a weak stimulation of $\mathrm{AT}_{4}$ would elicit BMPs and (2) enhanced the $\mathrm{AT}_{4}$-elicited synaptic input to $\mathrm{B} 31 / 32$ in cerebral and buccal ganglia dissected from trained animals.

For nerve stimulation, extracellular silver electrodes were placed on nerves $\mathrm{AT}_{4}$ and $\mathrm{En}_{2}$ (Fig. 1A), and a digital pulse generator (Model 1800; WPI) was used to deliver the stimuli.

\section{Classifications of BMPs}

The feeding CPG expresses BMPs, which can be associated with ingestion or rejection of food (Morton and Chiel 1993a,b). BMPs consist of specific patterns of neural activity, which represent cycles of protraction and retraction of the radula and odontophore. BMPs can be recorded from the buccal nerves $I_{2} n, R_{1}$, and $\mathrm{Bn}_{2,1}$. Large-unit activity in $\mathrm{I}_{2} \mathrm{n}$ (i.e., radula protraction) precedes large-unit activity in $\mathrm{Bn}_{2,1}$ (i.e., radula retraction), and large-unit activity in $\mathrm{Rn}_{1}$ (i.e., radula closure) overlaps to a varying extent with protraction and retraction activity (e.g., Cropper et al. 1990; Morton and Chiel 1993a,b; Nargeot et al. 1997; Kabotyanski et al. 2000). The large-unit activity in $\mathrm{Rn}_{1}$ corresponds to action potentials in the radula closure motor neuron B8, which has an axon in $\mathrm{Rn}_{1}$ (Morton and Chiel 1993b; Nargeot et al. 1999b).

As in previous studies (Morton and Chiel 1993a,b; Nargeot et al. 1997; Lechner et al. 2000b; Jing and Weiss 2001, 2002), we classified BMPs as ingestion-like if $\geq 50 \%$ of radula closure $\left(\mathrm{Rn}_{1}\right)$ activity occurred after the termination of the protraction $\left(\mathrm{I}_{2} \mathrm{n}\right)$ activity (Fig. 5A). The criterion for rejection-like BMPs was the occurrence of closure $\left(\mathrm{Rn}_{1}\right)$ activity during the protraction $\left(\mathrm{I}_{2} \mathrm{n}\right)$ activity, but no overlap between closure $\left(\mathrm{Rn}_{1}\right)$ and retraction $\left(\mathrm{Bn}_{2,1}\right)$ activity (Fig. 5B). BMPs that did not meet either of these two criteria were classified as other BMPs (Nargeot et al. 1997; Lechner et al. 2000b).

In the present study, only patterns (either spontaneous or evoked; see below) that consisted of activity in all three buccal nerves clustered in a complete protraction/retraction cycle were classified as BMPs. Patterns consisting of trains of activity in only one or two of the three nerves were classified as incomplete patterns and were not included in the study.

\section{Procedures for In Vitro Training}

The procedure for in vitro classical conditioning closely followed the in vivo training used by Lechner et al. (2000a). The number of training trials and the intertrial interval (ITI) were identical to those used in the behavioral studies. Paired, unpaired, and USalone protocols were used. In all of the protocols, electrical stimulation of $\mathrm{AT}_{4}(8 \mathrm{sec}, 5 \mathrm{~Hz}, 0.5-\mathrm{msec}$ pulses) served as the CS. The duration of $\mathrm{AT}_{4}$ electrical stimulation matched the duration of the tactile stimulation of the lips used in behavioral training (Lechner et al. 2000a). Electrical stimulation of $\mathrm{En}_{2}(4 \mathrm{sec}, 10 \mathrm{~Hz}$, 0.5 -msec pulses) served as the US. In the paired protocol, the CS preceded the US with an interstimulus interval (ISI) of $4 \mathrm{sec}$ (Fig. 1B1), and the CS and the US overlapped for $4 \mathrm{sec}$. In the unpaired procedure, the ISI between the CS and the US was 2 min (Fig. 1B2). An additional control group received only the US (Fig. 1B3). In all protocols, training consisted of 10 trials with a 4-min ITI (Fig. 1C).

Once the extracellular electrodes were in place, the high divalent ASW was exchanged for normal ASW. Preparations were rinsed for $10 \mathrm{~min}$ in ASW, and then the stimulus intensities for $\mathrm{En}_{2}$ and $\mathrm{AT}_{4}$ were adjusted. The intensity (typically 7-12 V) for stimulation of $\mathrm{En}_{2}$ was adjusted so that each US reliably elicited a BMP. $\mathrm{AT}_{4}$ appears to mediate aspects of both the CS and US because touching food to the lips can elicit a bite in vivo (Lechner et al. 2000a; see above). However, tactile stimulation of the lips alone (i.e., without food) in vivo is ineffective at eliciting bites prior to conditioning (Lechner et al. 2000a). Therefore, the overlapping functional consequences of nerve stimulation were minimized by selecting an in vitro stimulus for the CS that did not reliably elicit BMPs prior to training. Specifically, the intensity used for $\mathrm{AT}_{4}$ electrical stimulation was adjusted for each preparation so that the CS induced sporadic large-unit activity in the buccal nerves, but was not sufficient to elicit a BMP. From a pilot study, we estimated this intensity to be $5-10 \mathrm{~V}$. This finding was confirmed in the first series of experiments (see below), in which the average intensity of $\mathrm{AT}_{4}$ electrical stimulation (i.e., the in vitro CS) was $8.9 \pm 0.7 \mathrm{~V}(n=39)$.

Once the stimulus intensities for $\mathrm{En}_{2}$ and $\mathrm{AT}_{4}$ were adjusted, baseline activity was recorded for $30 \mathrm{~min}$. This baseline recording monitored the occurrence of spontaneous BMPs. A BMP was considered spontaneous if it occurred in the absence of nerve stimulation (either US or CS) or if it occurred beyond 1 min of nerve stimulation. A BMP was considered elicited if it occurred within 1 min of nerve stimulation. The stimulus intensities of the US and CS were confirmed $10 \mathrm{~min}$ into the baseline period. If the intensity of $\mathrm{En}_{2}$ or $\mathrm{AT}_{4}$ stimulation did not fulfill the criteria described above (i.e., $\mathrm{En}_{2}$ stimulation eliciting BMPs and $\mathrm{AT}_{4}$ stimulation inducing sporadic nerve activity but not BMPs), the voltage was adjusted slightly, but the nerves were not stimulated again until the Pre-Test began (Fig. 1C). Stimulus intensities for the CS and US remained fixed for the remainder of the experiment.

After the 30-min baseline period, four CSs were delivered with an ISI of 1 min (Pre-Test; Fig. 1C). The number of BMPs occurring during a 1-min period following each CS was counted. The total observation time of the Pre-Test was 4 min. After a 10 -min rest, one of the three training protocols was delivered. 
Preparations were included if the US stimulation elicited a BMP in at least half of the 10 training trials, and $13 \%$ of the preparations failed to meet this criterion. After training, the preparations were allowed to rest for $60 \mathrm{~min}$, during which time the occurrence of spontaneous BMPs was monitored. Finally, four CSs were delivered with an ISI of 1 min (Post-Test; Fig. 1C). The number of BMPs occurring during a 1-min period following each CS was counted. The total observation time of the Post-Test was 4 min.

\section{Experiment 1: Fictive Feeding}

Experiment 1 used intact ganglia (i.e., not desheathed) to examine changes in the activity of the feeding CPG following paired, unpaired, and US-alone training. As in our previous behavioral studies of classical conditioning of feeding (Lechener et al. 2000a,b), we examined the learning-related plasticity of CSevoked fictive feeding by analyzing the difference in the number of CS-evoked BMPs in the three experimental groups. The difference in the number of CS-evoked BMPs was defined as the number of BMPs elicited by four CSs during the Post-Test minus the number of BMPs elicited by four CSs during the Pre-Test. Experiment 1 also examined the changes in the occurrence of spontaneous BMPs after training and the type of CS-evoked BMPs elicited during the Post-Test. To assess the reproducibility of counting and classifying the different types of BMPs, the data from Experiment 1 were scored by three individuals, two of whom were not aware of the experimental history (i.e., type of training protocol) of each preparation. Scored data were compared among the three observers by using Kendall's coefficient of concordance (Zar 1999). A coefficient of concordance (between 0 and 1) was computed for the change in the number of CS-evoked BMPs as well as for each type of BMP (i.e., ingestion, rejection, and other BMPs) evoked during the Post-Test. Each coefficient of concordance $(0.99$ for the change in the number of CS-evoked BMPs; 0.86 for ingestion-like BMPs; 0.78 for rejection-like BMPs; and 0.7 for other BMPs) indicated significant agreement among the three observers. These results also indicated that the count and classification of BMPs were sufficiently objective. Therefore, a single observer was used to analyze the change in the number of CSevoked BMPs in Experiments 2-5.

\section{Experiment 2: Neuron B31/32}

In Experiment 2, changes in the properties of B31/32 were examined. Procedures were identical to those above, except that the right buccal ganglion was desheathed and two large motor neurons (B1 and B2) were removed with sharp forceps to provide access to neuron B31/32 (Fig. 1A). This procedure had no obvious effects on activity within the CPG (Lechner et al. 2000b). Intracellular recordings were made from $\mathrm{B} 31 / 32$ in the right buccal ganglion using conventional single- or two-electrode currentclamp techniques. Glass microelectrodes were filled with $3 \mathrm{M}$ potassium acetate (resistance 10-20 $\mathrm{M} \Omega$ ) and connected to an Axoprobe-1A electrometer (Axon Instruments). Neuron B31/32 was identified by an antidromic action potential in response to stimulation of $\mathrm{I}_{2} \mathrm{n}$ and by its characteristic plateau-like potential with nonovershooting somatic action potentials during the protraction phase of a BMP (Susswein and Byrne 1988; Hurwitz et al. 1994; Nargeot et al. 1997; Susswein et al. 2002). The resting membrane potential, the input resistance, and the CS-evoked synaptic input to B31/32 were determined $10 \mathrm{~min}$ prior to the Pre-Test. For measurements of the input resistance, the membrane potential of B31/32 was current-clamped at $-80 \mathrm{mV}$, and the voltage responses to three hyperpolarizing current pulses $(-5 \mathrm{nA}, 5 \mathrm{sec}$, 30 -sec intervals) were averaged. The CS-evoked synaptic input to B31/32 consisted of a complex potential (CPSP) elicited by electrical stimulation of $\mathrm{AT}_{4}$ (Lechner et al. 2000b). The CS-evoked cPSP was elicited in B31/32 by presenting a CS (i.e., $8 \mathrm{sec}, 5 \mathrm{~Hz}$, 0.5 -msec pulses) while the membrane potential of B31/32 was current-clamped at $-80 \mathrm{mV}$. To quantify the magnitude of the synaptic input to B31/32, both the maximal depolarization and the integral of the CS-evoked cPSP (i.e., the area of the cPSP during the 8-sec duration of the CS) were measured while the membrane potential of B31/32 was current-clamped at $-80 \mathrm{mV}$. The area of the cPSPs was analyzed with the Image Tool software version 3.0 (University of Texas Health Science Center in San Antonio, TX). After the input resistance and the cPSP of B31/32 were measured, the neuron was released from current clamp. The resting membrane potential, the input resistance, and the CSevoked synaptic input of B31/32 were measured again $10 \mathrm{~min}$ prior to the Post-Test.

The changes in resting membrane potential, input resistance, peak amplitude, and area of the cPSPs as a result of training were all expressed as a percentage of those measured before the Pre-Test (i.e., percent Pre-Test).

\section{Experiment 3: Neuron B4/5}

In Experiment 3, the properties of B4/5 were examined before and after training. The procedures for preparing the ganglia were identical to those described for Experiment 2. B4/5 was impaled with two microelectrodes and identified by its relative position within the ganglion (Gardner 1971, 1977) as well as its characteristic bursting activity during a BMP (Susswein and Byrne 1988; Church and Lloyd 1994; Kabotyanski et al. 1998). The resting membrane potential and the input resistance of B4/5 were measured $10 \mathrm{~min}$ prior to the Pre-Test and Post-Test. The input resistance was measured by averaging the membrane responses to three pulses of injected hyperpolarizing current $(5 \mathrm{sec},-5 \mathrm{nA})$ while the cell was current-clamped at $-80 \mathrm{mV}$. Also, the ability of the CS to activate B4/5 was tested before and after the training. B4/5 responded to the CS with a train of action potentials that lasted only as long as the stimulus. The number of action potentials elicited in B4/5 during the 4 CSs was counted before (i.e., during the Pre-Test) and after (i.e., during the Post-Test) training. Because the CS consistently elicited overshooting action potentials in the soma of B4/5, it was necessary to use a shorter duration of the nerve stimulus to examine the CS-evoked synaptic input to $\mathrm{B} 4 / 5$. Therefore, a single 0.5 -msec stimulation of $\mathrm{AT}_{4}$ was delivered $10 \mathrm{~min}$ prior to the Pre-Test and Post-Test and the cPSP in $\mathrm{B} 4 / 5$ was recorded while the membrane potential of $\mathrm{B} 4 / 5$ was current-clamped at $-80 \mathrm{mV}$. The magnitude of the cPSPs in B4/5 was determined by measuring the peak amplitude and the area of the cPSP over a duration of $400 \mathrm{msec}$. The change in the resting membrane potential, the input resistance, the peak amplitude, and the area of the cPSPs in B4/5 as a result of training were all expressed as a percentage of those measured before the training.

\section{Experiments 4 and 5: Monosynaptic Connection From CBI-2 to B31/32 and CS-Evoked Responses in CBI-2}

Experiments 4 and 5 explored whether in vitro classical conditioning affected the monosynaptic connection from CBI-2 to B31/32, the CS-evoked spike activity in CBI-2, and the CS-evoked cPSP in CBI-2. The right buccal ganglion was desheathed as in Experiment 2 to expose B31/32. In addition, the region of the cerebral ganglion corresponding to the ventral side of the right M cluster (Ono and McCaman 1980; Rosen et al. 1991) was desheathed with fine forceps to expose the soma of commandlike interneuron CBI-2. CBI-2 was identified according to the location of its soma in the $\mathrm{M}$ cluster as well as its spontaneous IPSPs (Rosen et al. 1991). Also, the identification of CBI-2 was confirmed at the end of the experiment by checking the ability of the neuron to drive multiple BMPs, when a 100-sec depolarizing pulse (6-12 nA) was injected into the cell (Rosen et al. 1991; Hurwitz et al. 1999; Jing and Weiss 2001). Neurons that did not fulfill this criterion were discarded. Because of the small size and the rather inaccessible location of its soma, CBI-2 was impaled with a single electrode. A total of 29 preparations were trained in vitro with either paired $(n=14)$ or unpaired $(n=15)$ protocols. These preparations were used to test the effect of classical conditioning on the synapse from CBI-2 to B31/32 (Experiment 4) and on the CS-evoked response in CBI-2 (Experiment 5). In addition, Experiment 5 examined the effect of in vitro classical conditioning on the resting membrane potential and input resistance of CBI-2. The analysis of the difference in the number of CS-evoked BMPs was performed on the total population of 29 preparations, 
and the intrinsic properties of CBI-2 and its CS-evoked response were recorded from most of these 29 preparations (12 paired and 13 unpaired). However, we were able to record the magnitude of the synapse from CBI-2 to B31/32 in only 15 preparations (seven paired and eight unpaired) of the total group of preparations trained in vitro. The difficulty in maintaining simultaneous impalement of neurons CBI-2 and B31/32 throughout the duration of each experiment lowered the rate of success in this experiment.

The efficacy of the excitatory synapse from CBI-2 to B31/32 can be rather low (Sánchez and Kirk 2002). Therefore, in Experiment 4, a 1-sec train at $10 \mathrm{~Hz}$ consisting of 10 -msec pulses (i.e., 10 action potentials in CBI-2) was used to obtain a quantifiable summating synaptic response in B31/32. The membrane potential of B31/32 was current-clamped at $-80 \mathrm{mV}$. The strength of the connection from CBI-2 to B31/32 was tested before and after the training, by measuring the peak amplitude and the area of the PSP in B31/32 over a 1-sec duration. For each preparation, the change in the amplitude and area of the synapse from CBI- 2 to B31/32 as a result of training was expressed as a percentage of that measured before training.

In Experiment 5, the CS-evoked spike activity in CBI-2 and the CS-evoked cPSP in CBI-2 were examined before and after training. $\mathrm{AT}_{4}$ stimulation elicits a train of action potentials in the somata of CBI-2 (Mozzachiodi et al. 2000), presumably because of recruitment of cerebral sensory neurons (Rosen et al. 1979), which converge on the command-like interneurons, including CBI-2 (Rosen et al. 1991). We noticed that in response to the CS, CBI-2 exhibited a train of action potentials that outlasted the duration of the CS. Therefore, the CS-evoked action potentials in CBI-2 were counted during a 30 -sec period after the onset of each CS during the Pre-Test and Post-Test. The CS-evoked synaptic input to CBI-2 was tested by delivering a stimulus to $\mathrm{AT}_{4}$, which consisted of $1-\mathrm{sec}, 5-\mathrm{Hz}, 0.5-\mathrm{msec}$ pulses. This abbreviated stimulus (i.e., $1 \mathrm{sec}$ vs. $8 \mathrm{sec}$ ) was used to minimize the contribution of polysynaptic pathways to the CS-evoked PSP in CBI-2. This stimulus induced a train of five summating PSPs in CBI-2 (i.e., synaptic profile). The area of the synaptic profile over a 1-sec duration and the amplitude of the first PSP were measured 10 min prior to the Pre-Test and Post-Test while the membrane potential of CBI- 2 was current-clamped at $-80 \mathrm{mV}$.

Finally, the resting membrane potential and the input resistance of CBI-2 were measured 10 min prior to the Pre-Test and Post-Test. The input resistance of CBI-2 was measured by averaging the membrane responses to three hyperpolarizing current pulses ( $-5 \mathrm{nA}, 200 \mathrm{msec}$, 30-sec intervals) while the membrane potential of CBI- 2 was current-clamped at $-80 \mathrm{mV}$.

\section{Statistical Analysis}

In Experiment 1, which included three groups, the difference in the number of CS-elicited BMPs produced by the three different training protocols (paired, unpaired, and US-alone) was analyzed with the Kruskal-Wallis test $(H)$. When indicated, post hoc pairwise comparisons were performed using the nonparametric ana$\log$ to the Student-Newman-Keuls multiple range test ( $q$; Zar 1999). In addition, the Kruskal-Wallis test was performed to analyze the change in the number of spontaneous BMPs produced by the three different training protocols. The Kruskal-Wallis test was also used to compare the different types of BMPs (i.e., ingestionlike, rejection-like, or other BMPs) expressed in the buccal ganglia after paired, unpaired, or US-alone training.

In Experiments 2-5, which included only two groups (i.e., paired and unpaired), a Mann-Whitney test $(U)$ was used to analyze the difference in the number of CS-evoked BMPs produced by training. A Mann-Whitney test was used also to analyze the change in the peak amplitude and area of the CS-evoked cPSP in $\mathrm{B} 31 / 32$, B4/5, and CBI-2; the magnitude of the synaptic connection from CBI-2 to B31/32; and the intrinsic properties (i.e., resting membrane potential and input resistance) of B31/32, B4/5, and CBI-2. The Spearman rank correlation coefficient $r$ was used to analyze the occurrence of a correlation between two sets of data. Finally, the Fisher exact test was used to analyze the $2 \times 2$ contingency tables (Zar 1999) describing the effect of training on the spiking activity of B4/5 and CBI-2, respectively (i.e., Experiments 3 and 5). Statistics were performed using SigmaStat 2.0 (Jandel Scientific).

\section{ACKNOWLEDGMENTS}

We thank Susan J. Cushman and Gregg A. Phares for helpful comments on the manuscript, and Björn Brembs and Fredy D. Reyes for scoring the data. This work was supported by National Institute of Mental Health Grant MH 58321.

The publication costs of this article were defrayed in part by payment of page charges. This article must therefore be hereby marked "advertisement" in accordance with 18 USC section 1734 solely to indicate this fact.

\section{REFERENCES}

Abrams, T., Yovell, Y., Onyike, C., Cohen, J., and Jarrard, H. 1998. Analysis of sequence-dependent interaction between transient calcium and transmitter stimuli in activating adenylyl cyclase in Aplysia: Possible contribution to CS-US sequence requirement during conditioning. Learn. Mem. 4: 496-509.

Anderson, J.A. 1967. Patterns of response of neurons in the cerebral ganglion of Aplysia californica. Exp. Neurol. 19: 65-77.

Antonov, I., Antonova, I., Kandel, E.R., and Hawkins, R.D. 2001. The contribution of activity-dependent synaptic plasticity to classical conditioning in Aplysia. J. Neurosci. 21: 6413-6422.

Antonov, I., Antonova, I., Kandel, E.R., and Hawkins R.D. 2003. Activity-dependent presynaptic facilitation and Hebbian LTP are both required and interact during classical conditioning in Aplysia. Neuron 37: 135-147.

Balaban, P.M., Bravarenko, N.I., Maksimova, O.A., Nikitin, E, Ierusalimsky, V.N., and Zakharov, I.S. 2001. A single serotonergic modulatory cell can mediate reinforcement in the withdrawal network of the terrestrial snail. Neurobiol. Learn. Mem. 75: 30-50.

Bao, J.-X., Kandel, E.R., and Hawkins, R.D. 1998. Involvement of presynaptic and postsynaptic mechanisms in a cellular analog of classical conditioning at Aplysia sensory-motor neuron synapses in isolated cell culture. J. Neurosci. 18: 458-466.

Benjamin, P.R., Staras, K., and Kemenes, G. 2000. A system approach to the cellular analysis of associative learning in the pond snail Lymnaea. Learn. Mem. 7: 124-131.

Brembs, B., Lorenzetti, F.D., Reyes, F.D., Baxter, D.A., and Byrne, J.H. 2002. Operant reward learning in Aplysia: Neuronal correlates and mechanisms. Science 296: 1706-1709.

Buonomano, D.V. and Byrne, J.H. 1990. Long-term synaptic changes produced by a cellular analog of classical conditioning in Aplysia. Science 249: 420-423.

Byrne, J.H. 1987. Cellular analysis of associative learning. Physiol. Rev. 67: 329-439.

Byrne, J.H., Castellucci, V.F., and Kandel, E.R. 1978. Contribution of individual mechanoreceptor sensory neurons to defensive gill-withdrawal reflex in Aplysia. J. Neurophysiol. 41: 418-431.

Carew, T.J. and Sahley, C.L. 1986. Invertebrate learning and memory: From behavior to molecules. Annu. Rev. Neurosci. 9: 435-487.

Carew, T.J., Walters, E.T., and Kandel, E.R. 1981. Classical conditioning in a simple withdrawal reflex in Aplysia californica. J. Neurosci. 1: $1426-1437$

Church, P.J. and Lloyd, P.E. 1994. Activity of multiple identified motor neurons recorded intracellularly during evoked feeding-like motor programs in Aplysia. J. Neurophysiol. 72: 1794-1809.

Colwill, R.M., Goodrum, K., and Martin, A. 1997. Pavlovian appetitive discriminative conditioning in Aplysia californica. Anim. Learn. Behav. 25: $268-276$.

Cropper, E.C. and Weiss, K.R. 1996. Synaptic mechanisms in invertebrate pattern generation. Curr. Opin. Neurobiol. 6: 833-841.

Cropper, E.C., Kupfermann, I., and Weiss, K.R. 1990. Differential firing patterns of the peptide containing cholinergic motor neurons B15 and B16 during feeding behavior in Aplysia. Brain. Res. 522: $176-179$

Crow, T. 1988. Cellular and molecular analysis of associative learning and memory in Hermissenda. Trends Neurosci. 11: 136-147.

Crow, T. and Alkon, D.L. 1978. Retention of an associative behavioral change in Hermissenda. Science 201: 1239-1241.

Davis, W.J. and Gillette, R. 1978. Neural correlate of behavioral plasticity in command neurons of Pleurobranchaea. Science 199: $801-804$

Dubnau, J. and Tully, T. 2001. Functional anatomy: From molecule to memory. Curr. Biol. 11: R240-R243.

Elliott, C.J. and Susswein, A.J. 2002. Comparative neuroethology of 
feeding control in molluscs. J. Exp. Biol. 205: 877-896.

Evans, C.G. and Cropper, E.C. 1998. Proprioceptive input to feeding motor programs in Aplysia. J. Neurosci. 8: 8016-8031.

Fredman, S.M. and Jahan-Parwar, B. 1980. Processing of chemosensory and mechanosensory information in identifiable Aplysia neurons. Comp. Biochem. Physiol. A 66: 25-34.

Gandhi, C.C. and Matzel, L.D. 2000. Modulation of presynaptic action potential kinetics underlies synaptic facilitation of type B photoreceptors after associative conditioning in Hermissenda. J. Neurosci. 20: 2022-2035.

Gardner, D. 1971. Bilateral symmetry and interneuronal organization in the buccal ganglia of Aplysia. Science 173: 550-553.

- 1977. Interconnections of identified multiaction interneurons in buccal ganglia of Aplysia. J. Neurophysiol. 40: 349-361.

Getting, P.A. 1975. Tritonia swimming: Triggering of a fixed action pattern. Brain Res. 95: 128-133.

Glanzman, D.L. 1995. The cellular basis of classical conditioning in Aplysia californica-It's less simple that you think. Trends Neurosci. 18: 30-36.

Hawkins, R.D., Abrams, T.W., Carew, T.J., and Kandel, E.R. 1983. A cellular mechanism of classical conditioning in Aplysia: Activity-dependent amplification of presynaptic facilitation. Science 219: 400-405.

Hawkins, R.D., Kandel, E.R., and Siegelbaum, S.A. 1993. Learning to modulate transmitter release: Themes and variations in synaptic plasticity. Annu. Rev. Neurosci. 16: 625-665.

Hawkins, R.D., Greene, W., and Kandel, E.R. 1998. Classical conditioning, differential conditioning, and second-order conditioning of the Aplysia gill-withdrawal reflex in a simplified mantle organ preparation. Behav. Neurosci. 112: 636-645.

Hurwitz, I., Goldstein, R.S., and Susswein, A.J. 1994. Compartmentalization of pattern-initiation and motor functions in the B31 and B32 neurons of the buccal ganglia of Aplysia californica. J. Neurophysiol. 71: 1514-1527.

Hurwitz, I., Neustadter, D., Morton, D.W., Chiel, H.J., and Susswein, A.J. 1996. Activity patterns of the B31/32 pattern initiators innervating the I2 muscle of the buccal mass during normal feeding movements in Aplysia californica. J. Neurophysiol. 75: 1309-1326.

Hurwitz, I., Kupfermann, I., and Susswein, A.J. 1997. Different roles of neurons B63 and B34 that are active during the protraction phase of buccal motor programs in Aplysia californica. J. Neurophysiol. 78: $1305-1319$.

Hurwitz, I., Perrins, R., Xin, Y., Weiss, K.R., and Kupfermann, I. 1999 C-PR neuron of Aplysia has different effects on "feeding" cerebral interneurons, including myomodulin-positive CBI-12. J. Neurophysiol. 81: 521-534.

Hurwitz, I., Kupfermann, I., and Weiss, K.R. 2003. Fast synaptic connections from CBIs to pattern-generating neurons in Aplysia: Initiation and modification of motor programs. J. Neurophysiol. 89: $2120-2136$.

Jahan-Parwar, B. and Fredman, S.M. 1976. Cerebral ganglion of Aplysia: Cellular organization and origin of nerves. Comp. Biochem. Physiol. A 54: $347-357$.

Jing, J. and Weiss, K.R. 2001. Neural mechanisms of motor program switch in Aplysia. J. Neurosci. 21: 7349-7362.

. 2002. Interneuronal basis of the generation of related but distinct motor programs in Aplysia: Implications for current neuronal models of vertebrate intralimb coordination. J. Neurosci. 22: 6228-6238.

Kabotyanski, E.A., Baxter, D.A., and Byrne, J.H. 1997. Involvement of neuron $\mathrm{B} 4 / 5$ in biasing the buccal CPG toward ingestion-like fictive feeding in Aplysia. Soc. Neurosci. Abstr. 23: 1046.

- 1998. Identification and characterization of catecholaminergic neuron B65, which initiates and modifies patterned activity in the buccal ganglia of Aplysia. J. Neurophysiol. 79: 605-621.

Kabotyanski, E.A., Baxter, D.A., Cushman, S.J., and Byrne, J.H. 2000. Modulation of fictive feeding by dopamine and serotonin in Aplysia. J. Neurophysiol. 83: 374-392.

Kemenes, G., Staras, K., and Benjamin, P.R. 1997. In vitro appetitive classical conditioning of feeding response in the pond snail Lymnaea stagnalis. J. Neurophysiol. 78: 2351-2361.

. 2001. Multiple types of control by identified interneurons in a sensory-activated rhythmic motor pattern. J. Neurosci. 21: $2903-2911$.

Kemenes, I., Kemenes, G., Andrew, R.J., Benjamin, P.R., and O'Shea, M. 2002. Critical time-window for NO-cGMP-dependent long-term memory formation after one-trial appetitive conditioning. $J$. Neurosci. 22: 1414-1425.

Kirk, M.D. 1989. Premotor neurons in the feeding system of Aplysia californica. J. Neurobiol. 20: 497-512.

Kovac, M.P., Matera, E.M., Volk, P.J., and Davis, W.J. 1986. Food avoidance learning is accompanied by synaptic attenuation in identified interneurons controlling feeding behavior in
Pleurobranchaea. J. Neurophysiol. 56: 891-905

Kuslansky, B., Weiss, K.R., and Kupfermann, I. 1978. A neural pathway mediating satiation of feeding behavior in Aplysia. Behav. Biol. 23: 230-237.

1987. Mechanisms underlying satiation of feeding behavior of the mollusc Aplysia. Behav. Neural. Biol. 48: 278-303.

Lamprecht, R., Hazvi, S., and Dudai, Y. 1997. cAMP response element-binding protein in the amygdala is required for long- but not short-term conditioned taste aversion memory. J. Neurosci. 17: $8443-8450$.

Lechner, H.A., Baxter, D.A., and Byrne, J.H. 2000a. Classical conditioning of feeding behavior in Aplysia: I. Behavioral analysis. J. Neurosci. 20: 3369-3376.

2000b. Classical conditioning of feeding behavior in Aplysia: II. Neurophysiological correlates. J. Neurosci. 20: 3377-3386.

Lloyd, P.E., Kupfermann, I., and Weiss, K.R. 1988. Central peptidergic neurons regulate gut motility in Aplysia. J. Neurophysiol. 59: $1613-1626$

Lukowiak, K. and Sahley, C.L. 1981. The in vitro classical conditioning of the gill withdrawal reflex of Aplysia californica. Science 212: $1516-1518$.

Maksimova, O.A. and Balaban, P.M. 1984. Neuronal correlates of aversive learning in command neurons for avoidance behavior of Helix lucorum L. Brain Res. 292: 139-149.

Maren, S. 2001. Neurobiology of Pavlovian fear conditioning. Annu. Rev. Neurosci. 24: 897-931.

Menzel, R. and Müller, U. 1996. Learning and memory in honeybees: From behavior to neural substrates. Annu. Rev. Neurosci. 19: 379404.

Morgan, P.T., Jing, J., Vilim, F.S., and Weiss, K.R. 2001. Interneuronal and peptidergic control of motor pattern switching in Aplysia. J. Neurophysiol. 87: 49-61.

Morton, D.W. and Chiel, H.J. 1993a. In vivo buccal nerve activity that distinguishes ingestion from rejection can be used to predict behavioral transitions in Aplysia. J. Comp. Physiol. [A] 172: 17-32.

. 1993b. The timing of activity in motor neurons that produce radula movements distinguishes ingestion from rejection in Aplysia. J. Comp. Physiol. [A] 173: 519-536.

Moyer, J.R., Thompson, L.T., and Disterhoft, J.F. 1996. Trace eyeblink conditioning increases CA1 excitability in a transient and learning specific manner. J. Neurosci. 16: 5536-5546.

Mozzachiodi, R., Baxter, D.A., Cleary, L.J., and Byrne, J.H. 2000. Afferent inputs to cerebral-buccal interneuron 2 (CBI-2) and its synaptic connections to B31/32 in Aplysia. Soc. Neurosci. Abstr. 26: 450.

Murphy, G.G. and Glanzman, D.L. 1997. Mediation of classical conditioning in Aplysia californica by long-term potentiation of sensorimotor synapses. Science 278: 467-471.

Nargeot, R., Baxter, D.A., and Byrne, J.H. 1997. Contingent-dependent enhancement of rhythmic motor pattern: An in vitro analog of operant conditioning. J. Neurosci. 17: 8093-8105.

. 1999a. In vitro analog of operant conditioning in Aplysia. I. Contingent reinforcement modifies the functional dynamics of an identified neuron. J. Neurosci. 19: 2247-2260.

- 1999b. In vitro analog of operant conditioning in Aplysia. II. Modifications of the functional dynamics of an identified neuron contribute to motor pattern selection. J. Neurosci. 19: 2261-2272.

Nargeot, R., Baxter, D.A., Patterson, G.W., and Byrne, J.H. 1999c. Dopaminergic synapses mediate neuronal changes in an analog of operant conditioning. J. Neurophysiol. 81: 1983-1987.

Ono, L.K. and McCaman, R.E. 1980. Identification of additional histaminergic neurons in Aplysia: Improvement of single cell isolation techniques for in tandem physiological and chemical studies. Neuroscience 5: 835-840.

Perrins, R. and Weiss, K.R. 1996. A cerebral central pattern generator in Aplysia and its connections with buccal feeding circuitry. J. Neurosci. 16: $7030-7045$.

Plummer, M.R. and Kirk, M.D. 1990. Premotor neurons B51 and B52 in the buccal ganglia of Aplysia californica: Synaptic connections, effects on ongoing motor rhythms, and peptide modulation. J. Neurophysiol. 63: $539-558$.

Rose, S.P. 2000. God's organism? The chick as a model system for memory studies. Learn. Mem. 7: 1-17.

Rosen, S.C., Weiss, K.R., and Kupfermann, I. 1979. Response properties and synaptic connections of mechanoafferent neurons in cerebral ganglion of Aplysia. J. Neurophysiol. 42: 954-974.

Rosen, S.C., Weiss, K.R., Cohen, J.L., and Kupfermann, I. 1982. Interganglionic cerebral-buccal mechanoafferents of Aplysia: Receptive fields and synaptic connections to different classes of neurons involved in feeding behavior. J. Neurophysiol. 48: 271-288.

Rosen, S.C., Susswein, A.J., Cropper, E.C., Weiss, K.R., and Kupfermann, I. 1989. Selective modulation of spike duration by serotonin and the neuropeptides, FMRFamide, $\mathrm{SCP}_{\mathrm{B}}$, buccalin and myomodulin in 


\section{Mozzachiodi et al.}

different classes of mechanoafferent neurons in the cerebral ganglion of Aplysia. J. Neurosci. 9: 390-402.

Rosen, S.C., Teyke, T., Miller, M.W., Weiss, K.R., and Kupfermann, I. 1991. Identification and characterization of cerebral-to-buccal interneurons implicated in the control of motor programs associated with feeding in Aplysia. J. Neurosci. 11: 3630-3655.

Sahley, C.L. 1995. What we have learned from the study of learning in the leech. J. Neurobiol. 27: 434-445.

Sahley, C.L. and Crow, T. 1998. Invertebrate learning: Current perspectives. In Neurobiology of learning and memory (eds. J.L. Martinez Jr. and R.P. Kesner), pp. 177-209. Academic Press, New York.

Sahley, C.L., Martin, K.A., and Gelperin, A. 1990. Analysis of associative learning in the terrestrial mollusc Limax maximus. II. Appetitive learning. J. Comp. Physiol. [A] 167: 339-345.

Sahley, C.L., Boulis, M.N., and Schuman, B. 1994. Associative learning modifies the shortening reflex in the semi-intact leech Hirudo medicinalis: Effects of pairing, predictability and CS preexposure. Behav. Neurosci. 108: 340-346.

Sánchez, J.A.D. and Kirk, M.D. 2000. Short-term synaptic enhancement modulates ingestion motor programs of Aplysia. J. Neurosci. 20: RC85:1-7.

. 2001. Cerebral-buccal pathways in Aplysia californica: Synaptic connections, cooperative interneuronal effects and feedback during buccal motor programs. J. Comp. Physiol. [A] 187: 801-815.

. 2002. Ingestion motor programs of Aplysia are modulated by short-term synaptic enhancement in cerebral-buccal interneuron pathways. Invert. Neurosci. 4: 199-212.

Schafe, G.E., Nader, K., Blair, H.T., and LeDoux, J.E. 2001. Memory consolidation of Pavlovian fear conditioning: A cellular and molecular perspective. Trends Neurosci. 24: 540-546.

Schwarz, M. and Susswein, A.J. 1986. Identification of the neural pathway for reinforcement of feeding when Aplysia learn that food is inedible. J. Neurosci. 6: 1528-1536.

Staras, K., Kemenes, G., and Benjamin, P.R. 1999. Cellular traces of behavioral classical conditioning can be recorded at several specific sites in a simple nervous system. J. Neurosci. 19: 347-357.
Susswein, A.J. and Byrne, J.H. 1988. Identification and characterization of neurons initiating patterned activity in the buccal ganglia of Aplysia. J. Neurosci. 8: 2049-2061.

Susswein, A.J. and Schwarz, M. 1983. A learned change of response to inedible food in Aplysia. Behav. Neural. Biol. 39: 1-6.

Susswein, A.J., Weiss, K.R., and Kupfermann, I. 1984. Internal stimuli enhance feeding behavior in the mollusc Aplysia. Behav. Neural. Biol. 41: 90-95.

Susswein, A.J., Hurwitz, I., Thorne, R., Byrne, J.H., and Baxter, D.A. 2002. Mechanisms underlying fictive feeding in Aplysia: Coupling between a large neuron with plateau potentials activity and a spiking neuron. J. Neurophysiol. 87: 2307-2323.

Talk, A.C. and Matzel, L.D. 1996. Calcium influx and release from intracellular stores contribute differentially to activity-dependent neuronal facilitation in Hermissenda photoreceptors. Neurobiol. Learn. Mem. 66: 183-197.

Thompson, R.F. and Kim, J.J. 1996. Memory systems in the brain and localization of a memory. Proc. Natl. Acad. Sci. 93: 13438-13444.

Walters, E.T. and Byrne, J.H. 1983. Associative conditioning of single sensory neurons suggests a cellular mechanism for learning. Science 219: 405-408.

Warman, E.N. and Chiel, H.J. 1995. A new technique for chronic single-unit extracellular recording in freely behaving animals using pipette electrodes. J. Neurosci. Methods 57: 161-169.

Welzl, H., D'Adamo, P., and Lipp, H.P. 2001. Conditioned taste aversion as a learning and memory paradigm. Behav. Brain. Res. 125: $205-213$.

Xin, Y., Weiss, K.R., and Kupfermann, I. 1995. Distribution in the central nervous system of Aplysia of afferent fibers arising from cell bodies located in the periphery. J. Comp. Neurol. 359: 627-643.

Zar, J.H. 1999. Biostatistical analysis, 4th ed. Prentice Hall, Engelwood Cliffs, NJ.

Received June 20, 2003; accepted in revised form October 3, 2003.

\section{Learning \& Memory}




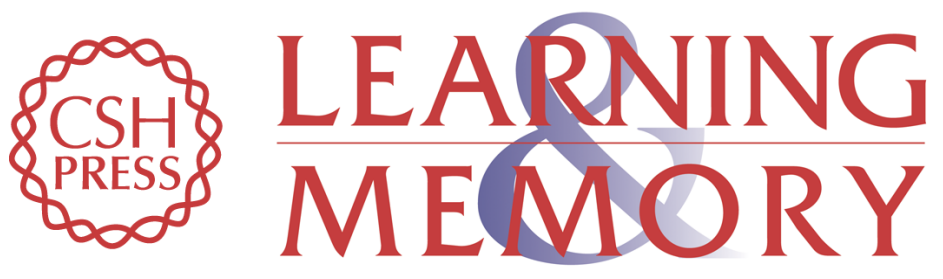

\section{In Vitro Analog of Classical Conditioning of Feeding Behavior in Aplysia}

Riccardo Mozzachiodi, Hilde A. Lechner, Douglas A. Baxter, et al.

Learn. Mem. 2003, 10:

Access the most recent version at doi:10.1101/lm.65303

References This article cites 93 articles, 35 of which can be accessed free at:

http://learnmem.cshlp.org/content/10/6/478.full.html\#ref-list-1

License

Email Alerting Receive free email alerts when new articles cite this article - sign up in the box at the Service top right corner of the article or click here. 